\title{
ON THE CHARACTER VARIETY OF THE THREE-HOLED PROJECTIVE PLANE
}

\author{
SARA MALONI AND FRÉDÉRIC PALESI
}

Abstract. We study the (relative) $\mathrm{SL}(2, \mathbb{C})$ character variety of the threeholed projective plane and the action of the mapping class group on it. We describe a domain of discontinuity for this action, which strictly contains the set of primitive stable representations defined by Minsky, and also the set of convex-cocompact characters.

\section{Contents}

1. Introduction

2. Notation

3. Analysis of $\boldsymbol{\omega}$-Markoff maps

4. Fibonacci growth and proof of Theorem A

5. Concluding remarks

Appendix A. Explicit expression of the $H$ function

Appendix B. Relation with the Torelli subgroup

Acknowledgment

References

\section{INTRODUCTION}

Given a finitely generated group $\Gamma$ and a semi-simple Lie group $G$, character varieties $\mathfrak{X}(\Gamma, G)$ are the (geometric invariant) quotients $\mathfrak{X}(\Gamma, G)=\operatorname{Hom}(\Gamma, G) / / G$

Received by the editors December 21, 2017, and, in revised form, July 18, 2019, November 11, 2019, December 16, 2019, and December 19, 2019.

2010 Mathematics Subject Classification. Primary 57M50, 20E05, 37A15.

Key words and phrases. Three-holed projective plane, character varieties, mapping class group, Bowditch set, domain of discontinuity.

The authors acknowledge support from U.S. National Science Foundation grants DMS 1107452, 1107263, 1107367 RNMS: "Geometric Structures and Representation Varieties" (the GEAR Network). This material is based upon work supported by the National Science Foundation under Grant No. 0932078000 while the authors were in residence at the Mathematical Sciences Research Institute in Berkeley, California, during the Spring 2015 semester. The authors are grateful to the organizers of the program for the invitations to participate, and to the MSRI and its staff for their hospitality and generous support.

The first author was partially supported by NSF grants DMS-1506920, DMS-1650811, DMS1839968 and DMS-1848346.

The second author was partially supported by the European Research Council under the European Community's seventh Framework Programme (FP7/2007-2013)/ERC grant agreement $n^{\circ}$ FP7-246918, and by ANR VALET (ANR-13-JS01-0010) and the work has been carried out in the framework of the Labex Archimede (ANR-11-LABX-0033) and of the A*MIDEX project (ANR11-IDEX-0001-02). 
of the spaces of representations $\rho: \Gamma \longrightarrow G$ from $\Gamma$ to $G$ up to conjugation, and have been extensively studied in many different aspects; see for example [6, 18. Here we will focus on the study of the action of the outer automorphism group $\operatorname{Out}(\Gamma)=\operatorname{Aut}(\Gamma) / \operatorname{Inn}(\Gamma)$ on $\mathfrak{X}(\Gamma, G)$ given by $\theta([\rho])=\left[\rho \circ \theta^{-1}\right]$. Our work is motivated by Fricke's classical result that the (orientation-preserving) mapping class group $\mathcal{M C G}(\Sigma)$ acts on the Teichmüller space $\mathcal{T}(\Sigma)$ of a closed orientable surface $\Sigma$ properly discontinuously. In fact, the Teichmüller space $\mathcal{T}(\Sigma)$ is one of the two connected components of the character variety $\mathfrak{X}\left(\pi_{1}(\Sigma), \mathrm{PSL}_{2}(\mathbb{R})\right)$ consisting of discrete and faithful representations from $\pi_{1}(\Sigma)$ into $\operatorname{PSL}_{2}(\mathbb{R})$, and $\mathcal{M C G}(\Sigma)$ is an index 2 subgroup (because of the orientation assumption) of the outer automorphism group Out $\left(\pi_{1}(\Sigma)\right)$. When $\Gamma$ is a word-hyperbolic group and $G$ has rank one, this result can be generalized by considering the action of $\operatorname{Out}(\Gamma)$ on the (open) set of convex-cocompact representations $\mathfrak{X}_{\mathrm{cc}}=\mathfrak{X}_{\mathrm{cc}}(\Gamma, G)$, which can be defined to be the representations $\rho: \Gamma \longrightarrow G$ for which the translation length of $\rho(\gamma)$ grows linearly in the cyclically reduced word length of $\gamma$ for all $\gamma \in \Gamma$. This result can be generalized further by considering the action on the set of Anosov representations; see $[3,12,15$.

When $\Gamma=\pi_{1}(\Sigma)$ is the fundamental group of a closed orientable surface $\Sigma$ and $G=\mathrm{PSL}_{2}(\mathbb{R})$ or $G=\mathrm{PSL}_{2}(\mathbb{C})$, Goldman [10] conjectured that the set $\mathfrak{X}_{\mathrm{cc}}\left(\pi_{1}(\Sigma), G\right)$ is the biggest domain of discontinuity for the $\mathcal{M C G}(\Sigma)$-action. So, conjecturally, the dynamical and the geometrical decomposition of the character varieties $\mathfrak{X}\left(\pi_{1}(\Sigma), G\right)$ coincide. On the other hand, if one considers surfaces $\Sigma_{g, b}$ with nonempty boundary, then the result is not true any longer and this is the case also for other hyperbolic groups; see [3 [5, 16, 17. The fundamental group $\pi_{1}\left(\Sigma_{g, b}\right)$ of surfaces $\Sigma_{g, b}$ with nonempty boundary is a free group $F_{n}$ and the mapping class group $\mathcal{M C G}\left(\Sigma_{g, b}\right)$ is a subgroup of $\operatorname{Out}\left(F_{n}\right)$. While the action of $\operatorname{Out}\left(F_{n}\right)$ on $\mathfrak{X}\left(F_{n}, \mathrm{PSL}_{2}(\mathbb{C})\right)$ is well known to be properly discontinuous on the set $\mathfrak{X}_{\mathrm{cc}}\left(F_{n}, \mathrm{PSL}_{2}(\mathbb{C})\right)$ of convex-cocompact (i.e., Schottky) characters, the action on the complement of these characters is more mysterious. Minsky [21] studied this action, and described the set of primitive-stable representations $\mathfrak{X}_{\mathrm{ps}}=\mathfrak{X}_{\mathrm{ps}}\left(F_{n}, \mathrm{PSL}_{2}(\mathbb{C})\right.$ )the ones for which the growth above is only requested for primitive elements. He, surprisingly, proved that $\mathfrak{X}_{\mathrm{ps}}$ is an open domain of discontinuity for the action of $\operatorname{Out}\left(F_{n}\right)$ which is strictly larger than the set of convex-cocompact (i.e., Schottky) characters.

In 2] Bowditch discussed a different method to study the character varieties $\mathfrak{X}\left(F_{n}, \mathrm{SL}(2, \mathbb{C})\right)$ and the action of $\mathcal{M C G}\left(\Sigma_{g, b}\right)$ on them. Bowditch studied the case $n=2$, but this approach was later generalized by Tan, Wong, and Zhang [24], by the authors and Tan [19], by Hu, Tan, and Zhang [13], and by Huang and Norbury [14, among others. They defined a domain of discontinuity $\mathfrak{X}_{Q}=$ $\mathfrak{X}_{Q}\left(\pi_{1}\left(\Sigma_{g, b}\right), \mathrm{SL}(2, \mathbb{C})\right)$, called the Bowditch set of representations, by considering representations $\rho: F_{n} \longrightarrow \mathrm{SL}(2, \mathbb{C})$ for which the growth discussed above is only required for elements $\gamma \in F_{n}$ representing simple closed curves in $\Sigma_{g, b}$. This subset of representations contains the set $\mathfrak{X}_{\mathrm{ps}}$, and hence is also strictly larger than the set of convex-cocompact characters. The properness of the action of $\mathcal{M C G}\left(\Sigma_{g, b}\right)$ on $\mathfrak{X}_{Q}$ follows from the fact that the set embeds in the set of proper functions $\mathcal{S}\left(\Sigma_{g, b}\right) \longrightarrow \mathbb{R}$ from the set of (free homotopy class of) simple closed curves $\mathcal{S}\left(\Sigma_{g, b}\right)$. On the other hand, the fact that $\mathfrak{X}_{Q}$ is open is much less obvious. Bowditch's idea was to use a combinatorial approach based on trace functions associated to simple 
closed curves, as we will explain below. In [19] the authors and Ser Peow Tan generalized those methods and studied the case $n=3$. In [19] we viewed the free group $F_{3}$ of rank three as the fundamental group of the four-holed sphere, while in this article we will consider $F_{3}$ as the fundamental group of the three-holed projective plane. In a recent work [20] joint with Yang we adapt the techniques used in 25] to the case of the thrice-punctured projective plane.

By a classical result on character varieties, see for example Fricke and Klein [7, the variety $\mathfrak{X}\left(F_{3}, \mathrm{SL}(2, \mathbb{C})\right)$ can be identified with the set of septuples $(a, b, c, d, x$, $y, z)$ in $\mathbb{C}^{7}$ such that:

(1) $a^{2}+b^{2}+c^{2}+d^{2}+a b c d=x(a b+c d)+y(b c+a d)+z(a c+b d)+4-x^{2}-y^{2}-z^{2}-x y z$, where $a=\operatorname{tr}(\rho(\alpha)), b=\operatorname{tr}(\rho(\beta)), c=\operatorname{tr}(\rho(\gamma)), d=\operatorname{tr}(\rho(\alpha \beta \gamma)), x=\operatorname{tr}(\rho(\alpha \beta))$, $y=\operatorname{tr}(\rho(\beta \gamma)), z=\operatorname{tr}(\rho(\alpha \gamma))$.
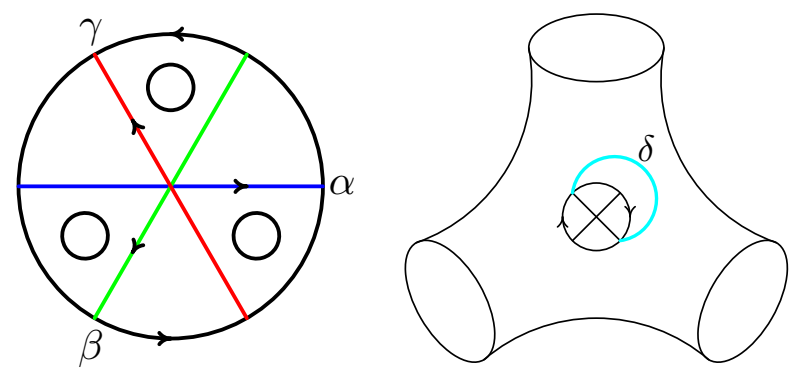

Figure 1. The space $N_{1,3}$ (in two different pictures) and the simple closed curves corresponding to $\alpha$ (in blue), $\beta$ (in green), $\gamma$ (in red), $\delta$ (in cyan).

In this paper we identify $F_{3}=\langle\alpha, \beta, \gamma, \delta \mid \alpha \beta \gamma \delta\rangle$ with the fundamental group of a three-holed projective plane $N$ so that $\alpha \beta, \beta \gamma, \alpha \gamma$ correspond to the three boundary components $\partial N$ of $N$; see Figure 1. In this case, the relative character variety $\mathfrak{X}_{(x, y, z)}(N)$, that is, the set of (classes of) representations for which the traces of the boundary curves are fixed, can be represented as quadruples $(a, b, c, d) \in \mathbb{C}^{4}$ which satisfy (11):

$$
\mathfrak{X}_{(x, y, z)}(N)=\left\{(a, b, c, d) \in \mathbb{C}^{4} \mid \text { equation (1) holds }\right\} .
$$

On the other hand, in [19] we identified $F_{3}$ to be the fundamental group of a fourholed sphere $S$, with the elements $\alpha, \beta, \gamma, \delta$ representing the peripheral simple closed curves of $\partial S$. In that case, the relative character variety $\mathfrak{X}_{(a, b, c, d)}(S)$ is identified with triples $(x, y, z) \in \mathbb{C}^{3}$ satisfying (11):

$$
\mathfrak{X}_{(a, b, c, d)}(S)=\left\{(x, y, z) \in \mathbb{C}^{3} \mid \text { equation (10) holds }\right\} .
$$

This hints that these two points of view are somehow "dual" to one another.

When considering $F_{3}$ as the fundamental group of the three-holed projective plane $N$ or of the four-holed sphere $S$, one can study the dynamics of the action of the (pure) mapping class groups $\mathcal{M C G}(N)$ and $\mathcal{M C G}(S)$, which are proper subgroups of Out $\left(F_{3}\right)$. In [19], the authors and Ser Peow Tan studied the action of $\mathcal{M C G}(S)$ on $\mathfrak{X}$ and described a domain of discontinuity $\mathfrak{X}_{Q}(S)$ which strictly contains $\mathfrak{X}_{\mathrm{ps}}$. More precisely, we studied the action of the group $\mathbb{Z}_{2} \star \mathbb{Z}_{2} \star \mathbb{Z}_{2}$ on the 
variety $\mathfrak{X}=\left\{(a, b, c, d, x, y, z) \in \mathbb{C}^{7} \mid\right.$ equation (1) holds $\}$ generated by the following involutions:

$$
\begin{aligned}
& \theta_{x}(a, b, c, d, x, y, z)=(a, b, c, d, p-y z-x, y, z), \\
& \theta_{y}(a, b, c, d, x, y, z)=(a, b, c, d, x, q-x z-y, z), \\
& \theta_{z}(a, b, c, d, x, y, z)=(a, b, c, d, x, y, r-x y-z),
\end{aligned}
$$

where

$$
p=a b+c d, \quad q=b c+a d, \quad r=a c+b d .
$$

The pure (orientation-preserving) mapping class group $\mathcal{M C G}(S)$ is an index 2 subgroup of the group $\mathbb{Z}_{2} \star \mathbb{Z}_{2} \star \mathbb{Z}_{2}$ defined by the above involutions. In fact, the mapping class group $\mathcal{M C G}(S)$ is generated by the three products of two distinct involutions. These involutions are defined by exchanging the two solutions of equation (11), considered as a quadratic equation in one of the variables $x, y$, or $z$, respectively.

In this article, we focus on the dynamics of $\mathcal{M C G}(N)$ on $\mathfrak{X}$. As in the case of the four-holed sphere, we actually study the action of $\mathbb{Z}_{2} \star \mathbb{Z}_{2} \star \mathbb{Z}_{2} \star \mathbb{Z}_{2}$ given by the following involutions:

$$
\begin{aligned}
& \theta_{a}(a, b, c, d, x, y, z)=(x b+z c+y d-b c d-a, b, c, d, x, y, z), \\
& \theta_{b}(a, b, c, d, x, y, z)=(a, x a+y c+z d-a c d-b, c, d, x, y, z), \\
& \theta_{c}(a, b, c, d, x, y, z)=(a, b, z a+y b+x d-a b d-c, d, x, y, z), \\
& \theta_{d}(a, b, c, d, x, y, z)=(a, b, c, y a+z b+x c-a b c-d, x, y, z) .
\end{aligned}
$$

The product of two of these involutions corresponds to a Dehn twist about a 2sided simple closed curve in $N$ (as it was the case for the four-holed sphere). This new point of view is very interesting. Among other reasons, it turns out that the Torelli subgroup $\mathcal{T}_{3}$ of $\operatorname{Out}\left(F_{3}\right)$ is an index 2 subgroup of the group generated by the seven involutions $\theta_{a}, \theta_{b}, \theta_{c}, \theta_{d}, \theta_{x}, \theta_{y}, \theta_{z}$, as we will prove in detail in Appendix B. See also Remark 2.4. We hope to combine these two approaches in a future paper, and study the action of the Torelli subgroup on the full character variety $\mathfrak{X}$.

The main result of this paper is the following.

Theorem A. There exists an open domain of discontinuity $\mathfrak{X}_{Q}(N)$ for the action of $\mathcal{M C G}(N)$ on $\mathfrak{X}$ which strictly contains $\mathfrak{X}_{\mathrm{ps}}$ (and so the set of discrete, faithful, convex-cocompact characters).

The set $\mathfrak{X}_{Q}(N)$ can be described as the set of representations $\rho \in \mathfrak{X}$ satisfying the following conditions:

(BQ1) for all $\gamma \in \mathcal{S}_{2}, \operatorname{tr} \rho(\gamma) \notin[-2,2]$; and

(BQ2) for all $K>0, \#\left\{\gamma \in \mathcal{S}_{2}|| \operatorname{tr} \rho(\gamma) \mid \leq K\right\}<\infty$,

where $\mathcal{S}_{2}$ is the set of free homotopy classes of unoriented 2 -sided simple closed curves in $N$. (The name (BQ) comes from Bowditch [2] who first defined the set $\mathfrak{X}_{Q}\left(\Sigma_{1,1}\right)$ for type-preserving representations $\rho: \pi_{1}\left(\Sigma_{1,1}\right) \longrightarrow \operatorname{SL}(2, \mathbb{C})$. The subindex Q comes from "quasi-Fuchsian space", as Bowditch conjectures that in the case of the once punctured torus the representations satisfying the (BQ) condition are exactly the quasi-Fuchsian ones.)

In Section 3.1 we will use another equivalent definition for $\mathfrak{X}_{Q}(N)$ which is more complicated to state, but is necessary to prove the main theorem. (The proof of the equivalence between the two definitions is contained in Section 4.2.) This 
equivalent definition is also useful if one wants to write a computer program which draws slices of the domain of discontinuity, because it requires checking condition (BQ2) for only one explicit value of $K$, and provides finite numerical certificates for this test. In addition, in Section 4.2 we will also prove that the set $\mathfrak{X}_{Q}(N)$ can be equivalently defined in terms of the growth of the elements of $\mathcal{S}_{2}$ in $N$. The elements of $\mathcal{S}$ can be arranged around a 4 -valent tree whose structure is respected by the mapping class group; see Section 2.5. In order to study the asymptotic growth of these elements, we use the Fibonacci function, a "comparison" function which can be defined recursively starting from the values around an edge of such a tree, and which is related to the word length of the elements of $\Gamma$ representing these simple closed curves; see Proposition 4.6. This will have as a corollary, the fact that $\mathfrak{X}_{Q}(N)$ contains the set $\mathfrak{X}_{\mathrm{ps}}$ of primitive-stable representations. On the other hand Proposition 4.13 shows that $\mathfrak{X}_{\mathrm{ps}} \subsetneq \mathfrak{X}_{Q}$. Proposition 4.12 shows that the Bowditch set $\mathfrak{X}_{Q}(N)$ can also be defined as

$$
\mathfrak{X}_{Q}(N)=\left\{\rho \in \mathfrak{X}\left(F_{3}\right)|\exists k=k(\rho),| \mathrm{L}(\rho(\gamma)) \mid \geq k \mathrm{~W}(\gamma) \forall \gamma \in \mathcal{S}(N)\right\},
$$

where $\mathcal{S}(N)$ is the set of (free homotopy classes of) nontrivial, nonperipheral simple closed curves in $N, \mathrm{~L}(\rho(\gamma))$ is the complex translation length of $\rho(\gamma)$, and $\mathrm{W}(\gamma)$ is the word length of $g$. This explains the connection with $\mathfrak{X}_{\mathrm{ps}}$ even more explicitly: $\mathfrak{X}_{Q}(N)$ is in fact defined by the same linear growth condition as $\mathfrak{X}_{\mathrm{ps}}$, but restricted to a certain subset of primitive elements (the simple closed curves) defined ad-hoc for the action of $\mathcal{M C G}(N)$ instead of the action of $\operatorname{Out}\left(F_{3}\right)$. See also Section 5.1 .

Sketch of the proof of Theorem A. The strategy to prove Theorem A consists mainly of a careful analysis using trace functions of simple closed curves in $N$, as previously done in Bowditch [2], Tan-Wong-Zhang [24] and Maloni-Palesi-Tan [19]. The main idea is to define a combinatorial complex $\Upsilon$, the dual of the complex of curves of $N$, and for any representation $\rho \in \mathfrak{X}$, assign an orientation on the 1 -skeleton of $\Upsilon$, and construct an attracting subtree on $\Upsilon$. Then, we prove that this attracting subtree is finite if and only if $\rho \in \mathfrak{X}_{Q}(N)$, and we use that to show that $\mathfrak{X}_{Q}(N)$ is open and the action of $\mathcal{M C G}(N)$ on it is properly discontinuous.

Plan of the paper. After setting up the notation and the required background that we need in Section 2 we describe the combinatorial viewpoint we adopt in Section 3, which uses trace functions on simple closed curves, and we describe the construction of the attracting subtree. We conclude in Section 4 after understanding the asymptotic growth of the length of simple closed curves. In the same section we also give different characterizations of the set $\mathfrak{X}_{Q}(N)$. In Appendix $\mathrm{A}$ we will prove an explicit formula related to the asymptotic growth of the representations along 2-sided simple closed curves, while in Appendix B we will prove that the Torelli subgroup $\mathcal{T}_{3}$ of $\operatorname{Out}\left(F_{3}\right)$ is an index 2 subgroup of the group generated by the seven involutions $\theta_{a}, \theta_{b}, \theta_{c}, \theta_{d}, \theta_{x}, \theta_{y}, \theta_{z}$.

\section{NotATion}

In this section we fix the notation which we will use in the rest of the paper and give some important definitions. Since in this article we will study representations $\rho: F_{3} \longrightarrow \mathrm{SL}(2, \mathbb{C})$ from the free group on three generators $F_{3}$ into $\mathrm{SL}(2, \mathbb{C})$, as we did in the article [19, we will follow the notation and structure of that paper. When possible, we will try to simplify the arguments, stating more clearly the 
relations between the different results and the ideas behind the proofs. Note that this work, as well as our previous work [19], are influenced by Bowditch's results [2], which were generalized by Tan, Wong, and Zhang [24. Note also that HuangNorbury [14 studied the particular case of the three-holed projective plane where all the boundary components are punctures, which makes equation (11) symmetric and much simpler. Their results go in a different direction from ours: we are interested in dynamical questions, while Huang and Norbury are more interested in some topological questions, as the study of a McShane identity, or of systoles of $N$. Again in the case when all the boundary components are punctures, our results are also related to the work of $\mathrm{Hu}$-Tan-Zhang [13.

2.1. Fundamental group $\Gamma$ of the three-holed projective plane $N$. Any (compact) nonorientable surface $N_{g, b}$ is characterized (topologically) by the number $g$ of cross-caps and the number $b$ of boundary components.

Let $N=N_{1,3}$ be a (topological) three-holed (real) projective plane, namely, a projective plane with three disjoint open disks removed, and let $\Gamma$ be its fundamental group. The group $\Gamma$ is isomorphic to the free group on three generators $\mathbb{Z} * \mathbb{Z} * \mathbb{Z}$ and admits the following presentation:

$$
\Gamma=\langle\alpha, \beta, \gamma, \delta \mid \alpha \beta \gamma \delta\rangle,
$$

where $\alpha, \beta, \gamma$, and $\delta$ are the loops described in Figure 1

Note that with these generators, the homotopy classes of the three boundary components, one for each removed disk, correspond to the elements $\alpha \beta, \beta \gamma$, and $\alpha \gamma$.

We define an equivalence relation $\sim$ on $\Gamma$ by: $g \sim h$ if and only if $g$ is conjugate to $h$ or $h^{-1}$. Then $\Gamma / \sim$ can be identified with the set of free homotopy classes of unoriented closed curves on $N$.

2.2. Simple closed curves on $N$. Let $\mathcal{S}=\mathcal{S}(N)$ be the set of free homotopy classes of essential simple closed curves on $N$. Recall that a curve is essential if it does not bound a disk, an annulus, or a Möbius strip. We will omit the word essential from now on. We can then identify $\mathcal{S}$ to be a well-defined subset of $\Gamma / \sim$.

Simple closed curves in a nonorientable surface are of two types: a simple closed curve is said to be 1-sided if its tubular neighborhood is homeomorphic to a Möbius strip, and 2-sided if the neighborhood is homeomorphic to an annulus. The 4 curves $\alpha, \beta, \gamma$, and $\delta$ described above are all 1 -sided. Let $\mathcal{S}_{i}$, where $i=1,2$, be the subset of $\mathcal{S}$ corresponding to $i$-sided simple closed curves. We recall that Dehn twists can only be defined along 2 -sided simple closed curves.

Remark 2.1. Since it will be important later, we note that in $N=N_{1,3}$, there is a one-to-one correspondence between:

- (unordered) pairs $\{\alpha, \beta\}$ of (free homotopy classes of) 1-sided simple closed curves intersecting exactly once; and

- (free homotopy classes of) 2-sided simple closed curves $\xi_{\alpha, \beta}$.

We will say that $\xi_{\alpha, \beta}$ is associated with the pair $\{\alpha, \beta\}$.

Proof. In fact, the $R$-neighborhood of any pair $\{\alpha, \beta\}$ of 1 -sided simple closed curves intersecting once corresponds to an embedded two-holed projective plane $M$. One of the boundary components of $M$ is homotopic to a boundary component of $N$, and we denote by $\xi_{\alpha, \beta}$ the other boundary curve, which is an essential 2-sided 


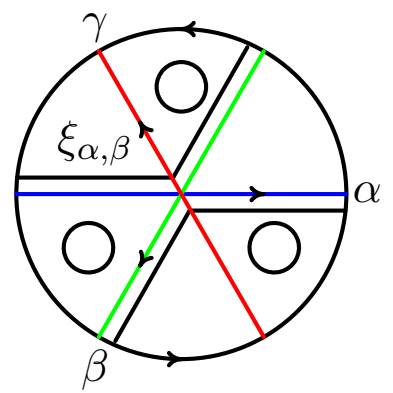

Figure 2. The curve $\xi_{\alpha, \beta}$ in black.

curve in $N$, and which corresponds to the element $\alpha \beta^{-1}$; see Figure 2, Conversely, any 2-sided essential simple closed curve on $N$ splits the surface into a pair of pants and a two-holed projective plane $M$ (note that there is a canonical choice of boundary component associated to any 2-sided curve), and there are exactly two 1-sided curves in $M$.

2.3. Relative character variety $\mathfrak{X}_{\boldsymbol{\omega}}(N)$. The character variety $\mathfrak{X}=\mathfrak{X}(\Gamma, \mathrm{SL}(2, \mathbb{C}))$ is the space of equivalence classes of representations $\rho: \Gamma \rightarrow \operatorname{SL}(2, \mathbb{C})$, where the equivalence classes are obtained by taking the closure of the orbit under the conjugation action by $\mathrm{SL}(2, \mathbb{C})$. As mentioned in the introduction, a classical result on the character varieties (see, for example, (9) on p. 298 of Fricke and Klein [7]) states that the map

$$
\begin{aligned}
& f: \mathfrak{X} \longrightarrow \mathbb{C}^{7} \\
& {[\rho] \bullet \longrightarrow\left(\begin{array}{l}
a \\
b \\
c \\
d \\
x \\
y \\
z
\end{array}\right)=\left(\begin{array}{c}
\operatorname{tr}(\rho(\alpha)) \\
\operatorname{tr}(\rho(\beta)) \\
\operatorname{tr}(\rho(\gamma)) \\
\operatorname{tr}(\rho(\delta)) \\
\operatorname{tr}(\rho(\alpha \beta)) \\
\operatorname{tr}(\rho(\beta \gamma)) \\
\operatorname{tr}(\rho(\alpha \gamma))
\end{array}\right) }
\end{aligned}
$$

provides an identification of $\mathfrak{X}$ with the set

$$
\left\{(a, b, c, d, x, y, z) \in \mathbb{C}^{7} \mid \text { equation (1) holds }\right\} .
$$

Let $\boldsymbol{\omega}=(x, y, z) \in \mathbb{C}^{3}$. Given fixed generators $\alpha, \beta, \gamma \in \Gamma$ of $\Gamma$, a representation $\rho: \Gamma \longrightarrow \mathrm{SL}(2, \mathbb{C})$ is said to be an $\boldsymbol{\omega}$-representation, or $\boldsymbol{\omega}$-character, if we have

$$
\begin{aligned}
& \operatorname{tr} \rho(\alpha \beta)=x, \\
& \operatorname{tr} \rho(\beta \gamma)=y, \\
& \operatorname{tr} \rho(\alpha \gamma)=z .
\end{aligned}
$$

The space of equivalence classes of $\boldsymbol{\omega}$-representations is denoted by $\mathfrak{X}_{\boldsymbol{\omega}}$ and is called the $\boldsymbol{\omega}$-relative character variety. These maps correspond to representations of the 
three-holed projective plane where we fix the conjugacy classes of the three boundary components in the space of closed orbits. The previous map gives an identification of $\mathfrak{X}_{\boldsymbol{\omega}}$ with the set

$$
\left\{(a, b, c, d) \in \mathbb{C}^{4} \mid \text { equation (1) holds }\right\} .
$$

2.4. The mapping class group $\mathcal{M C G}(N)$. The pure mapping class group $\mathcal{M C G}=$ $\mathcal{M C G}(N):=\pi_{0}(\operatorname{Homeo}(N))$ of $N$ is the subset of the group of isotopy classes of homeomorphisms of $N$ preserving each boundary component. Huang and Norbury gave a complete description of this group in [14, Section 2.7] and, in particular, they show that $\mathcal{M C G} \cong F \rtimes \operatorname{Stab}(\Delta)$, where $F$ is the group generated by the four involutions $\theta_{a}, \ldots, \theta_{d}$ defined in the introduction, $\Delta$ is the set $\{\alpha, \beta, \gamma, \delta\}$, and $\operatorname{Stab}(\Delta) \cong \mathbb{Z}_{2} \times \mathbb{Z}_{2}$. Since $F$ is a finite index subgroup of $\mathcal{M C G}(N)$, we will study its action on the character varieties $\mathfrak{X}$ and $\mathfrak{X}_{\boldsymbol{\omega}}$, as it is much simpler to describe.

We can now define the following property of words representing oriented simple closed curves, which will be important in the proof of Proposition 4.6. We say that a generating set $\left(u_{0}, u_{1}, u_{2}\right)$ of $\pi_{1}(N)$ is standard if

(a) $u_{0}, u_{1}, u_{2}$ and $u_{0} u_{1} u_{2}$ represent four 1 -sided simple loops intersecting pairwise once;

(b) the words $u_{i} u_{i+1}$ (indices modulo 3) represent the three peripheral loops;

(c) the words $u_{i} u_{i+1}^{-1}$ (indices modulo 3 ) represent three 2 -sided simple loops intersecting pairwise twice.

Remark 2.2. Up to the action of the mapping class group of $N$ and conjugation, there is only one standard generating set of $\pi_{1}(N)$.

Proof. A standard generating set gives rise to an unordered 4-tuple of 1-sided unoriented simple closed curves intersecting pairwise once. Such a 4 -tuple is unique up to the action of the mapping class group. To any three of the four curves, we can assign orientations such that appropriately chosen representatives $u_{0}, u_{1}, u_{2}$ in the corresponding conjugacy classes form (in any order) a standard generating set. This triple of orientations is itself unique only up to a full inversion of the orientations of the curves. The word $u_{0} u_{1} u_{2}$ can be assumed to represent the original fourth curve, up to applying an odd permutation or, equivalently, a full inversion of the orientations of the representatives $u_{0}, u_{1}, u_{2}$. This choice is compatible with condition (b) (or equivalently condition (c)). Indeed, from the correspondence described in Remark 2.1, a pair $(\alpha, \beta)$ of 1-sided simple curves intersecting once is associated to a two-holed projective plane $M$ whose two boundary components are homotopic to a boundary component of $N$ and to an essential 2-sided simple closed curve in $N$. For appropriately chosen representatives of $(\alpha, \beta)$, one of these boundary components corresponds to $\alpha \beta$, while the other corresponds to $\alpha \beta^{-1}$, and this pair of representatives is unique up to conjugation and inversion. So condition (b) gives rise to a triple of oriented 1-sided simple closed curves that is unique up to the action of the mapping class group, and representatives of that triple are unique up to conjugation. The picture for such a generating set is given in Figure 3, and one can check in the right picture that the three 2 -sided curves $u_{i} u_{i+1}^{-1}$ intersect pairwise twice.

Boundary slides are particular elements of the mapping class group of $N$ that are defined in the following way. An oriented separating 2-sided curve $\xi$ cuts 

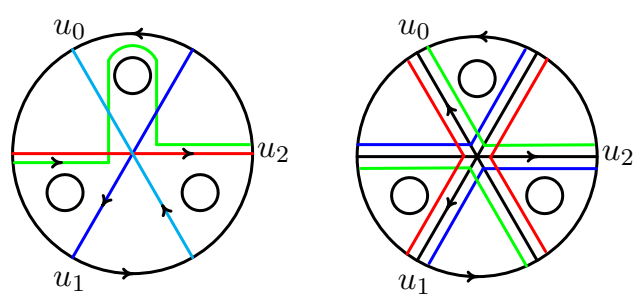

Figure 3. On the left, one can see the four 1-sided simple loops intersecting pairwise once: $u_{0}$ (cyan), $u_{1}$ (blue), $u_{2}$ (red), and $u_{1} u_{0} u_{2}$ (green). On the right, one can see the three 2 -sided simple loops intersecting pairwise twice: $u_{1} u_{0}^{-1}$ (red), $u_{2} u_{1}^{-1}$ (blue), and $u_{0} u_{2}^{-1}$ (green).

$N$ into a three-holed sphere and a one-holed Möbius strip (equivalently, a twoholed projective plane) $M$. In $M$ we can slide the hole along the unique 1-sided simple closed curve corresponding to the core of $M$ whose orientation is compatible with the orientation of $\xi$, giving rise to a diffeomorphism of $M$ fixing $\xi$. This diffeomorphism can be extended to $N$ by the identity, defining an element of the mapping class group of $N$, which we call a boundary slide associated to $\xi$. Given a standard generating set $\left(u_{0}, u_{1}, u_{2}\right)$, the action of a boundary slide associated to the 2 -sided simple closed curve $u_{1} u_{2}^{-1}$ can be expressed directly on the generating set; see Figure 4 . From the picture, one can see that the image of $\left(u_{0}, u_{1}, u_{2}\right)$ under the action of the boundaary slide is $\left(u_{2} u_{0} u_{1}, u_{2}^{-1}, u_{1}^{-1}\right)$. In Figure 4, one can see also the "new" fourth 1 -sided simple closed curve $u_{2} u_{0} u_{1} u_{2}^{-1} u_{1}^{-1}$ associated to this new standard generating set (drawn in green). Using the boundary slides associated to the other two 2 -sided simple curves $u_{2} u_{0}^{-1}$ and $u_{0} u_{1}^{-1}$, we can see that $\left(u_{1}^{-1}, u_{0}^{-1}, u_{1} u_{2} u_{0}\right)$ and $\left(u_{2}^{-1}, u_{0} u_{1} u_{2}, u_{1}^{-1}\right)$ are also standard generating sets. This proves the following result.

Remark 2.3. If $\left(u_{0}, u_{1}, u_{2}\right)$ is a standard generating set, then $\left(u_{2} u_{0} u_{1}, u_{2}^{-1}, u_{1}^{-1}\right)$, $\left(u_{1}^{-1}, u_{0}^{-1}, u_{1} u_{2} u_{0}\right)$, and $\left(u_{2}^{-1}, u_{0} u_{1} u_{2}, u_{1}^{-1}\right)$ are also standard generating sets.

Remark 2.4. The group generated by the seven involutions $\theta_{a}, \theta_{b}, \theta_{c}, \theta_{d}, \theta_{x}, \theta_{y}, \theta_{z}$ has a remarkable interpretation: it is a $\mathbb{Z}_{2}$ extension of the Torelli group $\mathcal{T}_{3}$ of the free group of rank three. Since this fact is of independent interest, we prove it in Appendix B.

2.5. The simplicial complex $\Upsilon$. In [19] we considered the countably infinite simplicial tree $\Sigma$ properly embedded in the plane all of whose vertices have degree 3 in order to define $\mu$-Markoff triples and $\mu$-Markoff maps. The complex $\Sigma$ is the dual to the Farey simplicial complex, which coincides with the complex of curves for the four-holed sphere $S$. We define here the analog for the three-holed projective plane case.

Let $\mathcal{C C}(N)$ be the complex of curves of $N$, which is the 3 -dimensional pure simplicial complex, where the $k$-cells are given by subsets of $k+1$ distinct (homotopy classes of ) 1 -sided simple closed curves in $N$ that pairwise intersect once. Scharlemann 23] proved that the 1-skeleton of $\mathcal{C C}(N)$ can be identified with the 1-skeleton of the cell complex formed from a tetrahedron by repeated stellar subdivision of the faces, but not the edges. 

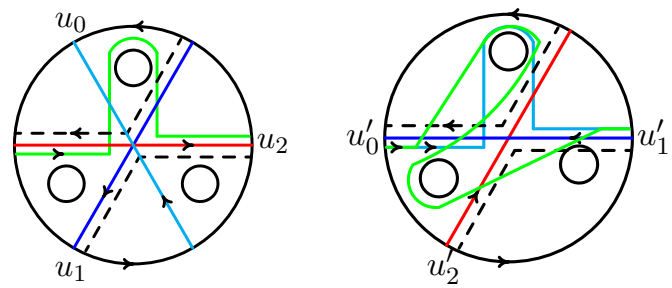

Figure 4 . On the left, you can see the original standard generating set $\left(u_{0}, u_{1}, u_{2}\right)$ with $u_{0}$ in cyan, $u_{1}$ in blue, $u_{2}$ in red, and $u_{0} u_{1} u_{2}$ in green, and the 2 -sided curve $u_{1} u_{2}^{-1}$ dashed in black. On the right, you can see the new generating set $\left(u_{0}^{\prime}, u_{1}^{\prime}, u_{2}^{\prime}\right)=$ $\left(u_{2} u_{0} u_{1}, u_{2}^{-1}, u_{1}^{-1}\right)$ obtained from $\left(u_{0}, u_{1}, u_{2}\right)$ under the boundary slides associated to the curve $u_{1} u_{2}^{-1}$ with $u_{0}^{\prime}=u_{0} u_{1} u_{2}$ in cyan, $u_{1}^{\prime}=u_{2}^{-1}$ in blue, $u_{2}^{\prime}=u_{1}^{-1}$ in red, and $u_{0}^{\prime} u_{1}^{\prime} u_{2}^{\prime}=u_{0} u_{1} u_{2} u_{1}^{-1} u_{2}^{-1}$ in green.

Let $\Upsilon$ be the dual to the simplicial complex $\mathcal{C C}(N)$. Let $\Upsilon^{(k)}$ denote the set of $k$-cells in $\Upsilon$. In particular from [23] we can see that $\Upsilon^{(1)}$ is a countably infinite simplicial tree all of whose vertices have degree 4 . We define a combinatorial distance on $\Upsilon^{(1)}$ by declaring that each edge has length 1 . Let $i(\cdot, \cdot)$ denote the geometric intersection number between two curves, that is, the minimal number of intersections in the homotopy classes of the curves. Note that the minimal intersection number between two 1 -sided curves in $N$, is 1 . We have the following sets:

- $\Upsilon^{(0)}=\left\{\left\{\alpha_{1}, \alpha_{2}, \alpha_{3}, \alpha_{4}\right\} \mid \alpha_{i} \in \mathcal{S}_{1}, i\left(\alpha_{i}, \alpha_{j}\right)=1\right.$ if $\left.i \neq j\right\}$;

- $\Upsilon^{(1)}=\left\{\left\{\alpha_{1}, \alpha_{2}, \alpha_{3}\right\} \mid \alpha_{i} \in \mathcal{S}_{1}, i\left(\alpha_{i}, \alpha_{j}\right)=1\right.$ if $\left.i \neq j\right\}$;

- $\Upsilon^{(2)}=\left\{\left\{\alpha_{1}, \alpha_{2}\right\} \mid \alpha_{i} \in \mathcal{S}_{1}, i\left(\alpha_{1}, \alpha_{2}\right)=1\right\}=\left\{\xi \in \mathcal{S}_{2}\right\}$;

- $\Upsilon^{(3)}=\left\{\alpha \in \mathcal{S}_{1}\right\}$.

So each vertex (resp., edge, face, or region) corresponds to a quadruple (resp., triple, pair, or singleton) of homotopy classes of 1-sided simple closed curves pairwise intersecting minimally. Notice that, thanks to Remark 2.1. each face in $\Upsilon^{(2)}$ also corresponds to a 2 -sided curve.

Note that, provided that the edges are long enough, one can embed $\Upsilon$ in $\mathbb{H}^{3}$ in such a way that each vertex has a full tetrahedral symmetry and the bisector of each edge is a plane of reflectional symmetry. In this way the involutions $\theta_{a}, \ldots, \theta_{d}$ act by hyperbolic isometries preserving $\Upsilon$.

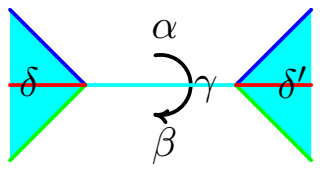

Figure 5. The edge $e \leftrightarrow\left(\alpha, \beta, \gamma ; \delta, \delta^{\prime}\right)$. (The coloring will be explained in the next section.)

We use greek letters $\alpha, \beta, \gamma, \alpha_{i}, \ldots$ to denote the elements of $\Upsilon^{(3)}$, while for the elements in $\Upsilon^{(2)}$ we use $\xi, \xi_{\alpha, \beta}, \ldots$ For an edge $e \in \Upsilon^{(1)}$, we also use the notation 
$e \leftrightarrow\left(\alpha, \beta, \gamma ; \delta, \delta^{\prime}\right)$ to indicate that $e=\alpha \cap \beta \cap \gamma$ and $e \cap \delta$ and $e \cap \delta^{\prime}$ are the endpoints of $e$; see Figure 5 .

2.6. The coloring of the tree. We choose a coloring of the regions and edges, namely a map $\mathcal{C}: \Upsilon^{(3)} \cup \Upsilon^{(1)} \longrightarrow\{1,2,3,4\}$ such that for any edge $e \leftrightarrow\left(a, \beta, \gamma ; \delta, \delta^{\prime}\right)$ we have $\mathcal{C}(e)=\mathcal{C}(\delta)=\mathcal{C}\left(\delta^{\prime}\right)$ and such that $\mathcal{C}(e), \mathcal{C}(\alpha), \mathcal{C}(\beta)$, and $\mathcal{C}(\gamma)$ are all different. The coloring is completely determined by a coloring of the four regions around any specific vertex, and hence is unique up to a permutation of the set $\{1,2,3,4\}$. We denote by $\Upsilon_{i}^{(3)}$ the set of regions with color $i$, and by $\Upsilon_{i}^{(1)}$ the set of edges with color $i$. In Figure 5 the edge $e=\left(\alpha, \beta, \gamma ; \delta, \delta^{\prime}\right) \in \Upsilon_{4}^{(1)}$ is drawn. (We didn't color the three regions $\alpha, \beta, \gamma$ around $e$.)

In the following, when $\alpha, \beta, \gamma, \delta$ are regions around a vertex, we will use the convention that $\alpha \in \Upsilon_{1}^{(3)}, \beta \in \Upsilon_{2}^{(3)}, \gamma \in \Upsilon_{3}^{(3)}$, and $\delta \in \Upsilon_{4}^{(3)}$, or that $\alpha_{i} \in \Upsilon_{i}^{(3)}$.

Remark 2.5. Note that there is a one-to-one correspondence between faces in $\Upsilon^{(2)}$ and so-called bi-colored geodesics in $\Upsilon^{(1)}$, namely maximal subtrees whose edges are of two colors. Such a set of edges is the boundary of a unique face.

In the same way, there is a one-to-one correspondence between faces in $\Upsilon^{(3)}$ and tri-colored subtrees in $\Upsilon^{(1)}$. Note also that tri-colored subtrees in $\Upsilon^{(1)}$ identify with (planar) Farey trees of a four-holed sphere as described in [19], since cutting $N$ along a 1-sided loop $g$ creates a four-holed sphere whose boundary curves are the three curves in $\partial N$ and the curve corresponding to $g^{2}$.

2.7. $\boldsymbol{\omega}$-Markoff quads. For a triple $\boldsymbol{\omega}=(x, y, z) \in \mathbb{C}^{3}$, an $\boldsymbol{\omega}$-Markoff quad is an ordered quadruple $\left(a_{1}, a_{2}, a_{3}, a_{4}\right)$ of complex numbers satisfying the $\boldsymbol{\omega}$-Markoff equation:

$$
\begin{aligned}
a_{1}^{2} & +a_{2}^{2}+a_{3}^{2}+a_{4}^{2}+a_{1} a_{2} a_{3} a_{4}=\lambda_{12}\left(a_{1} a_{2}+a_{3} a_{4}\right) \\
& +\lambda_{23}\left(a_{2} a_{3}+a_{1} a_{4}\right)+\lambda_{13}\left(a_{1} a_{3}+a_{2} a_{4}\right)+4-\lambda_{12}^{2}-\lambda_{23}^{2}-\lambda_{13}^{2}-\lambda_{12} \lambda_{23} \lambda_{13},
\end{aligned}
$$

or, equivalently,

$$
\sum_{i=1}^{4} a_{i}^{2}+\prod_{i=1}^{4} a_{i}=4-\lambda_{12} \lambda_{23} \lambda_{13}+\sum_{i<j=1}^{4} \lambda_{i j}\left(a_{i} a_{j}-\frac{\lambda_{i j}}{2}\right),
$$

where

$$
\begin{aligned}
& \lambda_{12}=\lambda_{34}=x, \\
& \lambda_{23}=\lambda_{14}=y, \\
& \lambda_{13}=\lambda_{24}=z .
\end{aligned}
$$

It is easily verified that, if $\left(a_{1}, a_{2}, a_{3}, a_{4}\right)$ is an $\boldsymbol{\omega}$-Markoff quad, then the quad obtained by replacing $a_{i}$ by $a_{i}^{\prime}$ is also an $\boldsymbol{\omega}$-Markoff quad, where

$$
a_{i}^{\prime}=\sum_{j \neq i} \lambda_{i j} a_{j}-\prod_{j \neq i} a_{j}-a_{i} .
$$

Since equation (5) is not symmetric in the variables $a_{1}, \ldots, a_{4}$ when $\boldsymbol{\omega}$ and hence $\lambda_{i, j}$ are fixed, then the quads obtained from permuting the entries are not necessarily $\boldsymbol{\omega}$-Markoff quads. This is a generalization of the situation analyzed in Huang-Norbury [14, where the equation defining the case of parabolic boundary components is symmetric, so much simpler to manipulate. 
We can see that there is a direct relation between $\boldsymbol{\omega}$-Markoff quads and $\boldsymbol{\omega}$ representations given by the following remark.

Remark 2.6. For any $\{\alpha, \beta, \gamma\} \in \Upsilon^{(1)}$ and for any $\boldsymbol{\omega} \in \mathbb{C}^{3}$, a representation $\rho$ is in $\mathfrak{X}_{\boldsymbol{\omega}}$ if and only if the quadruple $(\operatorname{tr}(\rho(\alpha)), \operatorname{tr}(\rho(\beta)), \operatorname{tr}(\rho(\gamma)), \operatorname{tr}(\rho(\alpha \beta \gamma)))$ is an $\boldsymbol{\omega}$-Markoff quad.

Using this correspondence, we can now see that the elementary operations on $\boldsymbol{\omega}$ Markoff quads defined in (7) are intimately related with the action of the mapping class group on the character of an $\boldsymbol{\omega}$-representation; see equation (3).

2.8. $\boldsymbol{\omega}$-Markoff maps. An $\boldsymbol{\omega}$-Markoff map is a function $\psi: \Upsilon^{(3)} \cup \Upsilon^{(2)} \longrightarrow \mathbb{C}$ such that the following properties hold:

(i) Vertex equation: $\forall v=\left\{\alpha_{1}, \ldots, \alpha_{4}\right\} \in \Upsilon^{(0)}$ (i.e., the $\alpha_{i} \in \Upsilon_{i}^{(3)}$ are the four regions meeting the vertex $v)$, the quad $\left(\psi\left(\alpha_{1}\right), \psi\left(\alpha_{2}\right), \psi\left(\alpha_{3}\right), \psi\left(\alpha_{4}\right)\right)$ is an $\boldsymbol{\omega}$-Markoff quad;

(ii) Edge equation: $\forall e=\left(\alpha_{i}, \alpha_{j}, \alpha_{k} ; \alpha_{l}, \alpha_{l}^{\prime}\right) \in \Upsilon_{l}^{(1)}$

$$
\psi\left(\alpha_{l}\right)+\psi\left(\alpha_{l}^{\prime}\right)=\lambda_{i l} \psi\left(\alpha_{i}\right)+\lambda_{j l} \psi\left(\alpha_{j}\right)+\lambda_{k l} \psi\left(\alpha_{k}\right)-\psi\left(a_{i}\right) \psi\left(a_{j}\right) \psi\left(a_{k}\right) ;
$$

(iii) Face equation: $\forall f=\left\{\alpha_{i}, \alpha_{j}\right\} \in \Upsilon^{(2)}$

$$
\psi\left(\alpha_{i}\right) \psi\left(\alpha_{j}\right)=\lambda_{i j}+\psi\left(\xi_{\alpha_{i}, \alpha_{j}}\right),
$$

where $\lambda_{i j}$ are defined in equation (6).

We shall use $\boldsymbol{\Psi}_{\boldsymbol{\omega}}$ to denote the set of all $\boldsymbol{\omega}$-Markoff maps and lower case letters to denote the $\psi$ values of the regions, that is, $\psi\left(\alpha_{i}\right)=a_{i}$.

Note that if the vertex equation (i) is satisfied at one vertex, then the edge equation (ii) guarantees that the vertex equation (i) is in fact satisfied at every vertex. This is related to the fact that the edge equations arise from the action of the (pure) mapping class group $\mathcal{M C G}(N)$ of the three-holed projective plane $N$, which preserves the boundary traces, and hence the relative character variety $\mathfrak{X}_{\boldsymbol{\omega}}$. This tells us the following.

Remark 2.7. If we fix one vertex in $\Upsilon^{(0)}$, we get a bijective correspondence between $\boldsymbol{\omega}$-Markoff maps and $\boldsymbol{\omega}$-Markoff quads. Now a choice of a vertex in $\Upsilon^{(0)}$, together with a coloring, can be translated into an ordered set of generators for $\pi_{1}(\Sigma)$. Hence, using Remark 2.6, there exists a bijective correspondence between the set $\boldsymbol{\Psi}_{\boldsymbol{\omega}}$ of $\boldsymbol{\omega}$-Markoff maps and the $\boldsymbol{\omega}$-relative character variety $\mathfrak{X}_{\boldsymbol{\omega}}$.

Given a Markoff map $\psi \in \boldsymbol{\Psi}_{\boldsymbol{\omega}}$, we now introduce a secondary function $\sigma=$ $\sigma_{\psi}: \Upsilon^{(2)} \rightarrow \mathbb{C}$ as follows. If $\xi=\left\{\alpha_{i}, \alpha_{j}\right\} \in \Upsilon^{(2)}$, then

$$
\sigma(\xi)=\left(a_{i}^{2}+a_{j}^{2}+\lambda_{i j}^{2}-a_{i} a_{j} \lambda_{i j}-4\right)\left(\lambda_{i k}^{2}+\lambda_{j k}^{2}+\psi(\xi)^{2}-\lambda_{i k} \lambda_{j k} \psi(\xi)-4\right),
$$

where $k \neq i$ and $k \neq j$. (From equation (5) we can see that any of the two possible choices for $k$ gives the same function.) The zeros of this function are representations which, restricted to a certain subsurface, are reducible, as we will explain in more detail in Remark 4.11. In Section 3.3.1, we will show that when for a certain face $\xi \in \Upsilon^{(2)}$ we have $\sigma(\xi)=0$, then the Markoff map $\psi$ is not a proper function. This is the reason behind Definition 3.3 
2.9. Orientation on $\Upsilon^{(1)}$. As in the previous papers 2, 19, 24, an $\boldsymbol{\omega}$-Markoff map $\psi \in \mathbf{\Psi}_{\boldsymbol{\omega}}$ determines an orientation on the 1 -skeleton $\Upsilon^{(1)}$ as follows. Suppose $e=\left(\alpha, \beta, \gamma ; \delta, \delta^{\prime}\right)$. If $|d|>\left|d^{\prime}\right|$, then the arrow on $e$ points towards $\delta^{\prime}$, while if $\left|d^{\prime}\right|>|d|$, then the arrow on $e$ points towards $\delta$. If $|d|=\left|d^{\prime}\right|$, then we choose the orientation of $e$ arbitrarily. The choice does not affect the arguments in the latter part of this paper. If the arrow on $e=\left(\alpha, \beta, \gamma ; \delta, \delta^{\prime}\right)$ points towards $\delta^{\prime}$, we will denote the oriented edge $e=\left(\alpha, \beta, \gamma ; \delta \longrightarrow \delta^{\prime}\right)$.

A vertex with at least two arrows pointing away from it is called a (generalized) fork. Note that in a (generalized) fork we can have two, three, or four arrows pointing away from it. In the last case, this is also called a source (so a source is a generalized fork).

\section{ANALYSis OF $\boldsymbol{\omega}$-MARKOFF MAPS}

In this section we define the so-called BQ-conditions for Markoff maps and analyze the behavior of maps satisfying them. In particular, for any Markoff map, using the orientation defined above, we will construct an attracting subtree, which we will use in the following section to prove Theorem A. In fact, the attracting subtree will be finite if and only if the Markoff maps satisfy the BQ-conditions.

3.1. BQ-conditions for Markoff maps. Let $\boldsymbol{\omega}=(x, y, z) \in \mathbb{C}^{3}$ and denote

$$
M=M(\boldsymbol{\omega})=\max \{|x|,|y|,|z|\} .
$$

Given $\psi \in \mathbf{\Psi}_{\boldsymbol{\omega}}$, and $K>0$, we define the subsets:

$$
\begin{aligned}
& \Upsilon_{\psi}^{(3)}(K)=\left\{\alpha \in \Upsilon^{(3)}|| \psi(\alpha) \mid<K\right\} \\
& \Upsilon_{\psi}^{(2)}(K)=\left\{\xi_{\alpha, \beta} \in \Upsilon^{(2)} \mid \alpha \text { or } \beta \in \Upsilon_{\psi}^{(3)}(K), \text { and }\left|\psi\left(\xi_{a, b}\right)\right|<K^{2}+M\right\} .
\end{aligned}
$$

The constant $K^{2}+M$ is chosen so that the following holds.

Lemma 3.1. If two adjacent regions $\alpha, \beta$ are in $\Upsilon_{\psi}^{(3)}(K)$, then the face $\xi_{\alpha, \beta}$ is in $\Upsilon_{\psi}^{(2)}(K)$

Proof. For example, if $|a|,|b|<K$, then

$$
|a b-x| \leq|a b|+|x|<(K)(K)+M=K^{2}+M .
$$

So in this case, $\xi_{\alpha, \beta} \in \Upsilon_{\psi}^{(2)}(K)$.

Definition 3.2 $\left(\mathrm{BQ}\right.$ set $\left.\left(\boldsymbol{\Psi}_{\boldsymbol{\omega}}\right)_{Q}\right)$. The Bowditch set $\left(\boldsymbol{\Psi}_{\boldsymbol{\omega}}\right)_{Q}$ is the set of $\boldsymbol{\omega}$-Markov maps $\psi \in \mathbf{\Psi}_{\boldsymbol{\omega}}$ such that the following conditions hold:

(BQ1) For all $\xi \in \Upsilon^{(2)}$, we have $\psi(\xi) \notin[-2,2]$.

(BQ3) For all $\xi \in \Upsilon^{(2)}$, we have $\sigma(\xi) \neq 0$.

(BQ4) The set $\Upsilon_{\psi}^{(2)}(2+M)$ is finite.

By Remark 2.7 we have a bijection $F_{\boldsymbol{\omega}}: \boldsymbol{\Psi}_{\boldsymbol{\omega}} \longrightarrow \mathfrak{X}_{\boldsymbol{\omega}}$ between the set $\boldsymbol{\Psi}_{\boldsymbol{\omega}}$ of $\boldsymbol{\omega}$ Markoff maps and the $\boldsymbol{\omega}$-relative character variety $\mathfrak{X}_{\boldsymbol{\omega}}$. So we can also define the set. 
Definition 3.3. Let $\left(\mathfrak{X}_{\boldsymbol{\omega}}\right)_{Q}$ be defined as $\left(\mathfrak{X}_{\boldsymbol{\omega}}\right)_{Q}:=F_{\boldsymbol{\omega}}\left(\left(\boldsymbol{\Psi}_{\boldsymbol{\omega}}\right)_{Q}\right)$. By abuse of notation, we will also call $\left(\mathfrak{X}_{\boldsymbol{\omega}}\right)_{Q}$ the Bowditch set. We define the set $\mathfrak{X}_{Q}$ of Bowditch representations, that will be referred to as the Bowditch set, as

$$
\mathfrak{X}_{Q}:=\bigcup_{\boldsymbol{\omega} \in \mathbb{C}^{3}}\left(\mathfrak{X}_{\boldsymbol{\omega}}\right)_{Q} .
$$

Remark 3.4. The definition of the BQ-condition for Markoff maps is different from the one given in the introduction. In particular, condition (BQ3) seems new, and condition (BQ4) is much weaker than condition (BQ2). However, in Proposition 4.10 we will see that the two definitions are, in fact, equivalent.

3.2. Connectedness. We start with the following key result.

Lemma 3.5 (Fork lemma). Let $\psi$ be an $\boldsymbol{\omega}$-Markoff map and $v=\{\alpha, \beta, \gamma, \delta\} \in \Upsilon^{(0)}$. Suppose that two arrows induced by $\psi$ point away from $v$. Then:

(1) At least one of the regions $\alpha, \beta, \gamma, \delta$ is in $\Upsilon_{\psi}^{(3)}(2+M)$.

(2) At least one of the six faces $\xi_{\alpha, \beta}, \xi_{\alpha, \gamma}, \xi_{\alpha, \delta}, \xi_{\beta, \gamma}, \xi_{\beta, \delta}, \xi_{\gamma, \delta}$ passing through $v$ is in $\Upsilon_{\psi}^{(2)}(2+M)$.

Proof. (1) Suppose, without loss of generality, that the outgoing arrows are in the direction opposite $\gamma$ and $\delta$. The edge relations give

$$
\begin{aligned}
& c+c^{\prime}=(x-a b) d+y b+z a, \\
& d+d^{\prime}=(x-a b) c+y a+z b,
\end{aligned}
$$

and the direction of arrows give

$$
|c| \geq\left|c^{\prime}\right| \text { and } \quad|d| \geq\left|d^{\prime}\right|
$$

From these, we get the two inequalities

$$
\begin{aligned}
& 2|c| \geq|a b-x||d|-(|y b|+|z a|), \\
& 2|d| \geq|a b-x||c|-(|y a|+|z b|),
\end{aligned}
$$

and adding both inequalities, we get:

$$
2(|c|+|d|) \geq|a b-x|(|c|+|d|)-(|a|+|b|)(|y|+|z|) .
$$

By contradiction, assume that $|a|,|b|,|c|,|d| \geq 2+M$. Then we have that $|a b-x| \geq$ $|a|+|b|$, and also that $|c|+|d|-|y|-|z| \geq 4$. So

$$
\begin{aligned}
2(|c|+|d|) & \geq|a b-x|(|c|+|d|-|y|-|z|), \\
|a b-x| & \leq \frac{2(|c|+|d|)}{|c|+|d|-|y|-|z|} \\
& =2+\frac{2(|y|+|z|)}{|c|+|d|-|y|-|z|} \\
& \leq 2+\frac{4 M}{4} \leq 2+M .
\end{aligned}
$$

On the other hand, if $|a b-x| \leq 2+M$, then $|a b| \leq 2+2 M$, and hence

$$
\min \{|a|,|b|\} \leq \sqrt{2+2 M}<2+M,
$$

which gives the contradiction proving (1).

(2) If two regions among $\alpha, \beta, \gamma$, and $\delta$ are in $\Upsilon_{\psi}^{(3)}(2+M)$, then by Lemma 3.1 the face at the intersection of these two regions is in $\Upsilon_{\psi}^{(2)}(2+M)$. 
So we can assume that only one region is in $\Upsilon_{\psi}^{(3)}(2+M)$. As the regions $\alpha, \beta$ and $\gamma, \delta$ play a different role, we have to distinguish two cases.

Case 1. $\gamma$ or $\delta$ is in $\Upsilon_{\psi}^{(3)}(2+M)$.

Without loss of generality, consider the case $|d|<2+M$. Assume by contradiction that $|a|,|b|,|c| \geq 2+M$. Then the edge relation $a b c=a y+b z+c x+d+d^{\prime}$ induces the inequality:

$$
(2+M)^{2}|\eta| \leq|a b c| \leq|y a|+|b z|+|c x|+2|d|<3 M|\eta|+4+2 M,
$$

where $|\eta|=\max \{|a|,|b|,|c|\}$. Hence we get $\left(4+M+M^{2}\right)|\eta|<4+2 M$, and so $|\eta|<\frac{1}{2}(M+2)$. This gives a contradiction. So at least one of $|a|,|b|$, or $|c|$ is less than $2+M$.

Case 2. $\alpha$ or $\beta$ is in $\Upsilon_{\psi}^{(3)}(2+M)$.

Without loss of generality, consider the case $|a|<2+M$. Assume that $\xi_{\alpha, \gamma}$ and $\xi_{\alpha, \delta}$ are not in $\Upsilon_{\psi}^{(2)}(2+M)$, so that $|a c-z| \geq(2+M)^{2}+M$ and $|a d-y| \geq(2+M)^{2}+M$. We will prove that, in this case, $\xi_{\alpha, \beta}$ is in $\Upsilon_{\psi}^{(2)}(2+M)$.

As $|c| \geq\left|c^{\prime}\right|$, and $|d| \geq\left|d^{\prime}\right|$, we have

$$
\begin{aligned}
& \left|a c^{\prime}-z\right| \leq|a c-z|+2 M, \\
& \left|a d^{\prime}-y\right| \leq|a d-y|+2 M,
\end{aligned}
$$

because $\left|a c^{\prime}-z\right| \leq\left|a c^{\prime}\right|+|z| \leq|a c|+|z| \leq|a c-z|+2|z|$. Hence we have

$$
\begin{aligned}
2|a c-z| & \geq|a c-z|+\left|a c^{\prime}-z\right|-2 M \\
& \geq\left|a\left(c+c^{\prime}\right)-2 z\right|-2 M \\
& =|a(-a b d+x d+y b+z a)-2 z|-2 M \\
& =\left|-(a b-x)(a d-y)+\left(a^{2}-2\right) z+x y\right|-2 M \\
& \geq|a b-x||a d-y|-\left|\left(a^{2}-2\right) z+x y\right|-2 M \\
& >|a b-x||a d-y|-\left(M^{3}+5 M^{2}+8 M\right) .
\end{aligned}
$$

Similarly we have $2|a d-y|>|a b-x||a c-z|-\left(M^{3}+5 M^{2}+8 M\right)$.

Adding the two inequalities, we obtain

$$
|a b-x|<2+\frac{2\left(M^{3}+5 M^{2}+8 M\right)}{|a c-z|+|a d-y|} .
$$

As $|a c-z|+|a d-y| \geq 2\left((2+M)^{2}+M\right)$, we get

$$
|a b-x|<2+\frac{2\left(M\left((2+M)^{2}+M\right)+4 M\right)}{2\left((2+M)^{2}+M\right)}<(2+M)^{2}+M .
$$

So $\xi_{\alpha, \beta} \in \Upsilon_{\psi}^{(2)}(2+M)$, as desired.

We say that a vertex is an $\varepsilon$-fork, if changing the value of one of the regions around $v$ by less than $\varepsilon$ makes it a fork. This means that $v$ has (at least two) arrows pointing away from it or pointing toward it, but such that the difference between the values at two neighboring regions, say $x, x^{\prime} \in \mathbb{C}$ are such that ||$x|-| x^{\prime}||<\varepsilon$.

Using this notion we get the following result.

Corollary 3.6. Let $\psi$ be an $\boldsymbol{\omega}$-Markoff map. There exists $\varepsilon_{0}>0$ such that for any $\varepsilon \in\left(0, \varepsilon_{0}\right)$ and any vertex $v \in \Upsilon^{(0)}$, if $v$ is an $\varepsilon$-fork, then the conclusions of Lemma 3.5 hold. 
Proof. The proof follows the same argument as in the fork lemma. Assume that $v$ is an $\varepsilon$-fork. We keep the same notation for $a, b, c, d, c^{\prime}, d^{\prime}$ as before, and without loss of generality we assume that

$$
|c| \geq\left|c^{\prime}\right| \text { and }|d| \geq\left|d^{\prime}\right|-\varepsilon .
$$

We assume that $|a|,|b|,|c|,|d| \geq 2+M$, and, using the same reasoning as before, we get that $|a b-x|<2+2 M+\frac{\varepsilon}{4}$, which implies that

$$
\min \{|a|,|b|\} \leq \sqrt{2+2 M+\frac{\varepsilon}{4}}
$$

There exists $\varepsilon_{1}$, depending only on $M$, such that for all $\varepsilon<\varepsilon_{1}$ we have $\sqrt{2+2 M+\frac{\varepsilon}{4}}$ $<2+M$. This means that we get a contradiction for all $\varepsilon<\varepsilon_{1}$. The important thing here is that the open inequality giving the contradiction only depends on $M$.

The second part is done in a similar way. In each case (Case 1 and Case 2) of the proof of Lemma 3.5 (2), the last inequality is open and depends only on $M$. So there exists a $\varepsilon_{2}$, depending only on $M$, such that the same contradiction arises. Finally, we set $\varepsilon_{0}=\min \left\{\varepsilon_{1}, \varepsilon_{2}\right\}$.

A consequence of the fork lemma is the following connectedness result.

Lemma 3.7. For all $\boldsymbol{\omega}$-Markoff maps $\psi$ and for all $K \geq 2+M$, the set $\Upsilon_{\psi}^{(3)}(K)$ is connected, that is, for any two regions $\alpha$ and $\alpha^{\prime}$ in $\Upsilon_{\psi}^{(2)}(K)$, there exists a sequence $\alpha_{0}=\alpha, \alpha_{1}, \ldots, \alpha_{k+1}=\alpha^{\prime}$ of regions in $\Upsilon_{\psi}^{(2)}(K)$ such that $\alpha_{j} \cap \alpha_{j+1} \neq \emptyset$ for all $j=0, \ldots, k$.

Proof. By contradiction, suppose that $\Upsilon_{\psi}^{(3)}(K)$ is not connected. Then there exist two regions $\delta, \delta^{\prime} \in \Upsilon_{\psi}^{(3)}(K)$ at distance $m \geq 1$ from each other, such that $\delta$ and $\delta^{\prime}$ cannot be connected within $\Upsilon_{\psi}^{(3)}(K)$, and such that the distance $m$ is minimal.

Case 1. $m=1$.

The regions $\delta$ and $\delta^{\prime}$ are connected by an edge $\left(\alpha, \beta, \gamma ; \delta, \delta^{\prime}\right)$ so that we have $d+d^{\prime}=-a b c+a y+b z+c x$. By hypothesis, $|a|,|b|,|c|>K$ and hence:

$$
\begin{aligned}
|a b c| & =|K+(|a|-K)||K+(|b|-K)||K+(|c|-K)| \\
& >K^{3}+K^{2}((|a|-K)+(|b|-K)+(|c|-K)) \\
& =K^{3}+K^{2}(|a|+|b|+|c|-3 K) .
\end{aligned}
$$

On the other hand,

$$
\begin{aligned}
|a b c| & \leq|a y|+|b z|+|c x|+|d|+\left|d^{\prime}\right| \\
& <M(|a|+|b|+|c|)+2 K \\
& \leq 3 K^{2}-4 K+(K-2)(|a|+|b|+|c|-3 K) \\
& <K(3 K-4)+K((|a|+|b|+|c|-3 K) \\
& <K^{3}+K(|a|+|b|+|c|-3 K),
\end{aligned}
$$

which gives a contradiction. So $m \neq 1$.

Case 2. $m>1$.

Consider the sequence of edges $\left(e_{i}\right)_{1 \leq i \leq m}$ going from $\delta$ to $\delta^{\prime}$. By minimality of $m$, the arrows $e_{1}$ and $e_{m}$ point towards the regions $\delta$ and $\delta^{\prime}$, respectively. Hence, one 
of the vertices along the sequence of edges is a fork. So, using Lemma 3.5(1), one of the regions neighboring the fork is in $\Upsilon_{\psi}^{(3)}(K)$, and this contradicts the minimality of $m$.

Lemma 3.8. For all $\boldsymbol{\omega}$-Markoff maps $\psi$ and for all $K \geq 2+M$, the set $\Upsilon_{\psi}^{(2)}(K)$ is connected, that is, for any two faces $\xi$ and $\xi^{\prime}$ in $\Upsilon_{\psi}^{(2)}(K)$, there exists a sequence $\xi_{0}=\xi, \xi_{1}, \ldots, \xi_{k+1}=\xi^{\prime}$ of faces in $\Upsilon_{\psi}^{(2)}(K)$ such that $\xi_{j} \cap \xi_{j+1} \neq \emptyset$ for all $j=0, \ldots, k$.

Proof.

Case 1. Assume the two faces $\xi=\{\alpha, \beta\}$ and $\xi^{\prime}=\left\{\alpha, \beta^{\prime}\right\}$ in $\Upsilon_{\psi}^{(2)}(K)$ are on the boundary of some region $\alpha \in \Upsilon_{\psi}^{(3)}(K)$.

Assume that $\xi$ and $\xi^{\prime}$ are not connected, and let $m$ be the minimal distance (measured along the tree) between the two connected components of $\Upsilon_{\psi}^{(2)}(K)$ containing $\xi$ and $\xi^{\prime}$. For convenience, we assume that this minimal distance is realized by the distance between $\xi$ and $\xi^{\prime}$. We distinguish three cases:

(1) $m=0$ : In this case, $\xi=\{\alpha, \beta\}$ and $\xi^{\prime}=\left\{\alpha, \beta^{\prime}\right\}$ share a common vertex, so $\left(\alpha, \beta, \beta^{\prime}\right)$ corresponds to an edge connecting $\xi$ to $\xi^{\prime}$.

(2) $m=1$ : In this case, $\xi=\{\alpha, \beta\}$ and $\xi^{\prime}=\left\{\alpha, \beta^{\prime}\right\}$ with an edge $\left(\alpha, \gamma, \delta ; \beta, \beta^{\prime}\right)$ between the two faces. The edge relation gives: $b+b^{\prime}=-a c d+x a+y c+z d$. So we get that

$$
\begin{aligned}
(a c-z)(a d-y) & =a(a c d-y c-z d)+y z \\
& =-a\left(b+b^{\prime}-x a\right)+y z \\
& =(x-a b)+\left(x-a b^{\prime}\right)-2 x+a^{2} x+y z .
\end{aligned}
$$

Hence

$$
\begin{aligned}
|a c-z||a d-y| & \leq|a b-x|+\left|a b^{\prime}-x\right|+2|x|+|a|^{2}|x|+|y||z| \\
& \leq 2\left(K^{2}+M\right)+2 M+K^{2} M+M^{2} \\
& \leq\left(K^{2}+M\right)^{2} .
\end{aligned}
$$

Hence one of the two faces $(\alpha, \gamma)$ or $(\alpha, \delta)$ is in $\Upsilon_{\psi}^{(2)}(K)$, which contradicts the minimality of $m$.

(3) $m>1$ : Consider the sequence of edges $\left(e_{i}\right)_{1 \leq i \leq m}$ going from $\xi$ to $\xi^{\prime}$. We claim that:

(a) $e_{1}$ is pointing towards $\xi$, and

(b) $e_{n}$ is pointing towards $\xi^{\prime}$.

In fact, without loss of generality, we can assume that $\xi=\{\alpha, \delta\}$ and the edge $e_{1}=\left(\alpha, \beta, \gamma ; \delta, \delta^{\prime}\right)$. If the edge $e_{1}$ pointed away from $\xi$, we would have $|d|>\left|d^{\prime}\right|$ and hence the face $\xi_{1}=\left\{\alpha, \delta^{\prime}\right\}$ would be in $\Upsilon_{\psi}^{(2)}(K)$ which contradicts the minimality of $m$. This shows the part (a) of the claim, and part (b) can be shown in a similar way. Following a similar argument as in Case 2 of Lemma 3.7, one of the vertices along the sequence is a fork. Hence using the fork Lemma 3.5 (2), one of the faces around this vertex is in $\Upsilon_{\psi}^{(2)}(K)$ which contradicts the minimality of $m$.

This proves the claim. 
Case 2. Let $\xi=\{\alpha, \beta\}$ and $\xi^{\prime}=\left\{\alpha^{\prime}, \beta^{\prime}\right\}$ be any face in $\Upsilon_{\psi}^{(2)}(K)$.

Then one of the regions $\alpha$ or $\beta$ is in $\Upsilon_{\psi}^{(3)}(K)$. Likewise, one of the regions $\alpha^{\prime}$ or $\beta^{\prime}$ is in $\Upsilon_{\psi}^{(3)}(K)$. So, without loss of generality we can assume that $\alpha$ and $\alpha^{\prime}$ are in $\Upsilon_{\psi}^{(3)}(K)$. As $K \geq 2+M$, the set $\Upsilon_{\psi}^{(3)}(K)$ is connected. Hence we can find a sequence of regions

$$
\alpha=\gamma_{0}, \gamma_{1}, \gamma_{2}, \ldots, \gamma_{n}=\alpha^{\prime} \text { in } \Upsilon_{\psi}^{(3)}(K)
$$

connecting $\alpha$ to $\alpha^{\prime}$. This gives in turn a sequence of faces $\xi_{k}=\left\{\gamma_{k}, \gamma_{k+1}\right\}$.

As $\left|\psi\left(\xi_{k}\right)\right| \leq\left|\psi\left(\gamma_{k}\right) \psi\left(\gamma_{k+1}\right)\right|+M \leq K^{2}+M$, it is clear that $\xi_{k}$ is in $\Upsilon_{\psi}^{(2)}(K)$. We can now use Case 1 to prove that two consecutive faces $\xi_{k}$ and $\xi_{k+1}$, which are on the boundary of the region $\gamma_{k+1}$, are connected, which ends the proof.

3.3. Escaping rays. Let $P$ be a geodesic arc in the tree $\Upsilon$, starting at vertex $v_{0} \in \Upsilon^{(0)}$ and consisting of edges $e_{n}$ joining $v_{n}$ to $v_{n+1}$. We say that such an infinite geodesic is an escaping ray if each edge $e_{n}$ is directed from $v_{n}$ towards $v_{n+1}$. First we will consider the behavior of the regions around a fixed face $\xi=\{\alpha, \beta\} \in \Upsilon^{(2)}$, and then we will describe the general case.

3.3.1. Neighbors of a face. For each face $\xi=\{\alpha, \beta\} \in \Upsilon^{(2)}$, its boundary is a bi-infinite path consisting of edges of the form $\left(\alpha, \beta, \gamma_{n} ; \delta_{n-1}, \delta_{n}\right)$ alternating with edges $\left(\alpha, \beta, \delta_{n} ; \gamma_{n}, \gamma_{n+1}\right)$, where $\left\{\alpha, \beta, \gamma_{n}, \delta_{n}\right\}$ and $\left\{\alpha, \beta, \gamma_{n+1}, \delta_{n}\right\}$ are vertices in $\Upsilon^{(0)}$. We say that the regions $\gamma_{n}$ and $\delta_{n}$ are the neighboring regions to the face $\xi$. The edge relations on two consecutive edges give:

$$
\begin{aligned}
c_{n+1} & =-(a b-x) d_{n}-c_{n}+(z a+y b) ; \\
d_{n+1} & =-(a b-x) c_{n+1}-d_{n}+(z b+y a) \\
& =(a b-x) c_{n}+\left((a b-x)^{2}-1\right) d_{n}+(z b+y a-(a b-x)(z a+y b)) .
\end{aligned}
$$

We can reformulate these equations in terms of matrices:

$$
\left(\begin{array}{c}
c_{n+1} \\
d_{n+1}
\end{array}\right)=\left(\begin{array}{cc}
-1 & -(a b-x) \\
(a b-x) & (a b-x)^{2}-1
\end{array}\right) \cdot\left(\begin{array}{l}
c_{n} \\
d_{n}
\end{array}\right)+\left(\begin{array}{c}
z a+y b \\
z b+y a-(a b-x)(z a+y b)
\end{array}\right) .
$$

Note that the setting is similar to the situation for the four-holed sphere in 19, up to a change of variables. See also Appendix A.

Let $\lambda \in \mathbb{C}$ such that $\lambda+\lambda^{-1}=(a b-x)^{2}-2$. It corresponds to the two eigenvalues of the matrix $\left(\begin{array}{cc}-1 & -(a b-x) \\ (a b-x) & (a b-x)^{2}-1\end{array}\right)$. Note that $|\lambda|=1$ if and only if $(a b-x) \in[-2,2]$. Take $\Lambda$ to be a square root of $\lambda$.

If $(a b-x) \in\{-2,2\}$, then the matrix is parabolic and the unique eigenvalue is $\lambda=1$. In the case $a b-x=2$, we can express the sequences $c_{n}$ and $d_{n}$ as:

$$
\begin{aligned}
& c_{n}=n^{2}((z a+y b)-(z b+y a))+n(z b+y a)-(2 n-1)\left(c_{0}+d_{0}\right), \\
& d_{n}=n^{2}((z b+y a)-(z a+y b))-n(z a+y b)+2 n\left(c_{0}+d_{0}\right) .
\end{aligned}
$$

The other case $a b-x=-2$ is similar.

If $(a b-x) \notin\{-2,2\}$, then we can express the sequences $c_{n}$ and $d_{n}$ as:

$$
\begin{aligned}
& c_{n}=A \Lambda^{2 n}+B \Lambda^{-2 n}+\eta(a b-x), \\
& d_{n}=-\left(A \Lambda^{2 n+1}+B \Lambda^{-2 n-1}\right)+\zeta(a b-x),
\end{aligned}
$$


where $\eta$ and $\zeta$ are two complex functions with parameters $a, b$ defined by:

$$
\begin{aligned}
& \eta(t)=\frac{1}{4-t^{2}}(2(z a+y b)-t(z b+y a)), \\
& \zeta(t)=\frac{1}{4-t^{2}}(2(z b+y a)-t(z a+y b)) .
\end{aligned}
$$

Note that $\eta(a b-x)$ and $\zeta(a b-x)$ are the coordinates of the center of the conic in coordinates $(c, d)$ defined by the vertex relation (11) (with parameters $(a, b, x, y, z))$.

The product $A B$ is given by the following:

$$
A B=\frac{1}{\left(4-(a b-x)^{2}\right)^{2}}\left(a^{2}+b^{2}+x^{2}-a b x-4\right)\left((a b-x)^{2}+y^{2}+z^{2}-y z(a b-x)-4\right) .
$$

Using the secondary function $\sigma$, it can be written more concisely as:

$$
A B=\frac{\sigma(\xi)}{\left(4-(\psi(\xi))^{2}\right)^{2}} .
$$

From this discussion, we deduce the following result.

Lemma 3.9. With the notations introduced above, we have

(1) If $\psi(\xi) \in(-2,2)$, then $\left|c_{n}\right|$ and $\left|d_{n}\right|$ remain bounded.

(2) If $\psi(\xi) \in\{-2,2\}$, then $\left|c_{n}\right|$ and $\left|d_{n}\right|$ grow at most quadratically.

(3) If $\psi(\xi) \notin[-2,2]$, and $\sigma(\xi) \neq 0$, then $\left|c_{n}\right|$ and $\left|d_{n}\right|$ grows exponentially as $n \rightarrow+\infty$ and as $n \rightarrow-\infty$.

(4) If $\psi(\xi) \notin[-2,2]$, and $\sigma(\xi)=0$, then $\left(c_{n}, d_{n}\right)$ converges to $(\eta(a b-x), \zeta(a b-$ $x)$ ) when $n \rightarrow+\infty$ or $n \rightarrow-\infty$.

Note that an escaping ray can't occur in case (3).

3.3.2. General escaping ray. Now we consider the general case.

Lemma 3.10. Suppose that $\left\{e_{n}\right\}_{n \in \mathcal{N}}$ is an escaping ray. Then:

- either the ray is eventually contained in some face $\xi \in \Upsilon^{(2)}$ such that $\psi(\xi) \in$ $[-2,2]$, or $\sigma(\xi)=0$,

- or the ray meets infinitely many elements $\gamma \in \Upsilon_{\psi}^{(2)}(2+M)$.

Proof. First, suppose that there exists $n_{0} \in \mathbb{N}$, and a face $\xi \in \Upsilon^{(2)}$ such that, for all $n \geq n_{0}$, the edges $e_{n}$ are contained in $\xi$. As the path is descending, it means that the values of the regions meeting $\xi$ at the edges $e_{n}$ stay bounded. Hence, from Lemma 3.9. we infer that, either $\psi(\xi) \in[-2,2]$, or $\sigma(\xi)=0$.

Now suppose that we are not in the first case, that is, the ray is not eventually contained in some face $\xi \in \Upsilon^{(2)}$. Then we prove the following.

Claim. There exists $n_{0} \in \mathcal{N}$ such that $e_{n_{0}}$ is contained in a face $\xi_{0} \in \Upsilon_{\psi}^{(2)}(2+M)$.

The proof is very similar to the one of the fork Lemma 3.5. Let $\varepsilon>0$. Let $\alpha_{i}, \beta_{i}, \gamma_{i}, \delta_{i}$ be the four sequences of regions corresponding to the four regions around the head of edge $e_{i}$. At least two sequences, say $\left(\left|c_{i}\right|\right)$ and $\left(\left|d_{i}\right|\right)$, among $\left(\left|a_{i}\right|\right),\left(\left|b_{i}\right|\right),\left(\left|c_{i}\right|\right),\left(\left|d_{i}\right|\right)$ are infinite, weakly decreasing, and bounded below. So for $n$ large enough, we have two consecutive directed edges $e_{n}=\left(\alpha, \beta, \gamma ; \delta^{\prime} \rightarrow \delta\right)$ and $e_{n+1}=\left(\alpha, \beta, \delta ; \gamma \rightarrow \gamma^{\prime}\right)$ with a common vertex $v_{n}$ and common face $\xi_{\alpha, \beta}$, and such that

$$
|d| \leq\left|d^{\prime}\right| \leq|d|+\varepsilon \quad \text { and } \quad\left|c^{\prime}\right| \leq|c| .
$$


This means that the vertex $v_{n}$ is an $\varepsilon$-fork. So for $\varepsilon$ small enough we can apply Corollary 3.6 and get that one of the regions around the vertex $v_{n}$ is in $\Upsilon_{\psi}^{(3)}(2+M)$. In turn this implies the existence of a face $\xi_{0}$ in $\Upsilon_{\psi}^{(2)}(2+M)$ around the edge $e_{n}$, which proves the claim.

As we are not in the first case, it means that there exists $n_{1}>n_{0}$ such that $e_{n_{1}} \notin \xi_{0}$. Let $v_{1}$ be the head of the arrow $e_{n_{1}}$ and consider the escaping ray starting at $v_{1}$. By the same reasoning, the ray will meet a face $\xi_{1} \in \Upsilon_{\psi}^{(2)}(K)$. By induction, this proves that the initial ray will eventually meet an infinite number of faces in $\Upsilon_{\psi}^{(2)}(2+M)$.

We will also need the following result, giving a necessary condition for a given element $\xi \in \Upsilon^{(2)}$ to satisfy $\sigma(\xi)=0$.

Lemma 3.11. Suppose that we have $\psi \in \mathbf{\Psi}_{\boldsymbol{\omega}}$ and $\xi \in \Upsilon^{(2)}$ such that $\sigma(\xi)=0$, and let $K>2+M$. Then at least one of the following is true:

- The face $\xi$ belongs to $\Upsilon_{\psi}^{(2)}(K)$.

- The set $\Upsilon_{\psi}^{(2)}(K)$ is infinite.

Proof. If $\psi(\xi) \in[-2,2]$, then the first condition is satisfied. So suppose that $\psi(\xi) \notin$ $[-2,2]$. Let $\gamma_{n}$ and $\delta_{n}$ be the two sequences of neighboring regions around the face $\xi$. From Lemma 3.9. (4) we can see that the sequence $\left(c_{n}, d_{n}\right)$ converges to a certain point in $\mathbb{C}^{2}$ when $n$ goes to infinity.

Let $\varepsilon>0$. For $n$ large enough, the successive values of $\left|c_{n}\right|$ and of $\left|d_{n}\right|$ are as close as we want. So the vertex $v_{n}$, which is the intersection $\xi \cap \gamma_{n} \cap \delta_{n}$ is an $\varepsilon$-fork. Hence for $\varepsilon$ small enough we can apply Corollary 3.6 and one of the faces containing $v_{n}$ is in $\Upsilon_{\psi}^{(2)}(K)$.

Let $\xi_{n}$ be a face containing $v_{n}$ and in $\Upsilon_{\psi}^{(2)}(K)$. If $\xi \notin \Upsilon_{\psi}^{(2)}(K)$, then the sequence $\xi_{n}$ consists of infinitely many distinct elements as $\xi$ is the only face that contains more than two vertices in the sequence $\left\{v_{n}\right\}$. So either $\xi$ is in $\Upsilon_{\psi}^{(2)}(K)$ or there exists an infinite number of faces in $\Upsilon_{\psi}^{(2)}(K)$.

3.4. Attracting subtree. In this section, for all $\psi \in \boldsymbol{\Psi}_{\boldsymbol{\omega}}$ and for all $K \geq 2+M$ we define an attracting subtree $T_{\psi}(K)$. A subtree $T$ of $\Upsilon^{(1)}$ is said to be $\psi$-attracting if all edges not in $T$ all point towards $T$ for the orientation on $\Upsilon^{(1)}$ given by $\psi$. In order to do that, first we construct, for all faces $\xi \in \Upsilon^{(2)}$, an attracting subarc in the boundary of $\xi$.

3.4.1. Attracting arc $J_{\psi}(K, \xi)$. Let $K \geq 2+M$. For each map $\psi \in \mathbf{\Psi}_{\boldsymbol{\omega}}$ and for each face $\xi \in \Upsilon_{\psi}^{(2)}(K)$, we will construct explicitly a connected nonempty subarc $J=J_{\psi}(K, \xi)$ such that the following conditions are satisfied:

(1) Every edge in $\xi$ that is not in $J$ points towards $J$.

(2) If $X \in \Upsilon_{\psi}^{(k)}(K)$, with $k=2,3$, and $\xi \cap X=e$, then $e \in J$.

From Lemma 3.9, we can define a function

$$
H_{\psi}: \Upsilon^{(2)} \longrightarrow \mathbb{R} \cup\{\infty\},
$$

with the following properties:

- If $\xi \in \Upsilon^{(2)}$, such that $\sigma_{\psi}(\xi)=0$ or $\psi(\xi) \in[-2,2]$, then we define $H_{\psi}(\xi)=$ $\infty$. 
- If $\xi=\xi_{\alpha, \beta} \in \Upsilon^{(2)}$, such that $\sigma_{\psi}(\xi) \neq 0$ and $\psi(\xi) \notin[-2,2]$, we have that, if $\left(\gamma_{n}\right)_{n \in \mathbb{Z}}$ and $\left(\delta_{n}\right)_{n \in \mathbb{Z}}$ are the sequences of neighboring regions around $\xi$, then there exist integers $n_{1}, n_{2}$ such that:

- $\left|c_{n}\right| \leq H_{\psi}(\xi)$ and $\left|d_{n}\right| \leq H_{\psi}(\xi)$ if and only if $n_{1} \leq n \leq n_{2} ;$

- $\left|c_{n}\right|$ and $\left|d_{n}\right|$ are monotonically decreasing for $n<n_{1}$, and increasing for $n>n_{2}$.

(See Section 3.3 for the definition of "neighboring regions".)

As the explicit expression of the function $H_{\psi}$ is not relevant in the following proofs, we will defer its definition to Appendix A.

Remark 3.12. Note that, for any fixed face $\xi \in \Upsilon^{(2)}$, the function

$$
H .(\xi): \Psi_{\boldsymbol{\omega}} \longrightarrow \mathbb{R} \cup\{\infty\}
$$

defined by $\psi \mapsto H_{\psi}(\xi)$ is continuous over the Bowditch set.

The arc formed by the union of the edges $e_{n}$ for $n_{1} \leq n \leq n_{2}$ satisfies property (1). In order for it to satisfy property (2), we need to slightly modify the function $H$ into

$$
H_{\psi}^{*}: \mathbb{R} \times \Upsilon^{(2)} \longrightarrow \mathbb{R} \cup\{\infty\}
$$

as follows:

$$
H_{\psi}^{*}\left(K, \xi_{\alpha, \beta}\right):=\max \left\{H_{\psi}(\xi), \frac{K^{2}+2 M}{\min \{|a|,|b|\}}\right\}
$$

Now we define:

$$
J_{\psi}(K, \xi):=\bigcup_{\gamma \in \Upsilon^{(3)}\left(H_{\psi}^{*}(K, \xi)\right)} \gamma \cap \xi .
$$

Indeed, if one of the faces $\xi_{\alpha, \gamma}$ or $\xi_{\beta, \gamma}$ is in $\Upsilon_{\psi}^{(2)}(K)$, then the region $\gamma$ belongs to $\Upsilon_{\psi}^{(3)}\left(\frac{K^{2}+2 M}{\min \{|a|,|b|\}}\right) \subset \Upsilon_{\psi}^{(3)}\left(H_{\psi}^{*}(\xi, K)\right)$ and so $\xi \cap \xi_{\alpha, \beta}=\gamma \cap \xi_{\alpha, \beta} \in J$. Similarly, if $\gamma \in \Upsilon_{\psi}^{(3)}(K)$, then, since $K<\frac{K^{2}+2 M}{\min \{|a|,|b|\}}$, we have $\gamma \in \Upsilon_{\psi}^{(3)}\left(H_{\psi}^{*}(K, \xi)\right)$ and so $\gamma \cap \xi_{\alpha, \beta} \in J$.

This is a subset of the edges in the boundary of $\xi$ such that the regions corresponding to each edge have image less than $H_{\psi}^{*}(K, \xi)$. Note that, if a face $\xi \in \Upsilon^{(2)}$, satisfies $\sigma_{\psi}(\xi)=0$ or $\psi(\xi) \in[-2,2]$, then the subset $J_{\psi}(H, \xi)$ is the entire face $\xi$ because $H_{\psi}(\xi)=\infty$.

Now it is clear that the arc $J_{\psi}(K, \xi)$ constructed by this procedure satisfies conditions (1) and (2) above.

\subsubsection{Attracting subtree $T_{\psi}(K)$. Let $\psi \in \mathbf{\Psi}_{\boldsymbol{\omega}}$.}

The previous discussion guarantees the existence of an attracting subarc for all faces $\xi \in \Upsilon_{\psi}^{(2)}(K)$. Hence, we can construct the set:

$$
T_{\psi}(K)=\bigcup_{\xi \in \Upsilon_{\psi}^{(2)}(K)} J_{\psi}(K, \xi) .
$$

This set is a union of edges, and we can prove the following result.

Proposition 3.13. The set $T_{\psi}(K)$ is connected and $\psi$-attracting. 
Proof. Denote $T=T_{\psi}(K)$, and let $e$ and $e^{\prime}$ be two edges in $T$. From the construction of $T$, there are two faces $\xi, \xi^{\prime} \in \Upsilon_{\psi}^{(2)}(K)$ such that $e \in \xi$ and $e^{\prime} \in \xi^{\prime}$. By edge connectedness of $\Upsilon_{\psi}^{(2)}(K)$ proved in Lemma 3.8 we can find a sequence of faces $\xi=\xi_{0}, \xi_{1}, \ldots, \xi_{n}=\xi^{\prime}$ such that $\xi_{k} \cap \xi_{k+1}=e_{k} \neq \emptyset$. By property (2), each edge $e_{k}$ is in both $J_{\psi}\left(K, \xi_{k}\right)$ and $J_{\psi}\left(K, \xi_{k+1}\right)$, and so in $T_{\psi}(K)$. As $J_{\psi}\left(K, \xi_{k}\right)$ is connected, the edges $e_{k-1}$ and $e_{k}$ are connected inside $\xi_{k}$. So, we get that $e$ and $e^{\prime}$ are connected inside $T$.

Now we prove that $T$ is $\psi$-attracting, using the following claim.

Claim. The arrows in the circular neighborhood $C(T)$ around $T$ point towards $T$. (Recall that the the circular neighborhood $C(T)$ of a finite subtree $T \subset \Upsilon$ is the set of edges $e$ of $\Upsilon$ which intersect $T$, but which are not in $T$.)

Indeed, suppose that we have a vertex $v=\{\alpha, \beta, \gamma, \delta\} \in T$ and an edge $e \notin T$ pointing outward. One of the faces containing $v$ is in $\Upsilon_{\psi}^{(2)}(K)$, for example $\xi_{\alpha, \beta}$. By the property (1) of the arc $J_{\psi}\left(K, \xi_{\alpha, \beta}\right)$, it is clear that $e$ cannot be contained in $\xi_{\alpha, \beta}$. So we can assume, without loss of generality, that $e=\left(\beta, \gamma, \delta ; \alpha, \alpha^{\prime}\right)$.

If $|a|<K$, then we have $\left|a^{\prime}\right|<K$. Then by connectedness of $\Upsilon_{\psi}^{(3)}(K)$, one of the regions $\beta, \gamma$, or $\delta$ is also in $\Upsilon_{\psi}^{(3)}(K)$. So one of the faces $\xi_{\alpha^{\prime}, \beta}, \xi_{\alpha^{\prime}, \gamma}$, or $\xi_{\alpha^{\prime}, \delta}$ is in $\Upsilon_{\psi}^{(2)}(K)$. This contradicts the connectedness of $T$.

If $|a|>K$, then we have $|b|<K+\frac{2 M}{K}$. The edge relation gives

$$
|b c-y||b d-z|<2|a b|+|x y|+|x||b|^{2} .
$$

Hence using $(2+M) \leq K$ we obtain

$$
|b c-y||b d-z|<(2+M)^{3}+M^{2}+4 M<(2+M)^{4},
$$

which proves that one of the faces $\xi_{\beta, \gamma}$ or $\xi_{\beta, \delta}$ is in $\Upsilon_{\psi}^{(2)}(K)$, again contradicting the connectedness of $T$. This proves the claim.

Now, if there exists an arrow outside the tree that doesn't point towards the tree, then there exists a vertex $v$ at distance at least 1 of the tree that is a fork. Hence using the fork Lemma 3.5) (2) one of the faces containing $v$ is in $\Upsilon_{\psi}^{(2)}(K)$ which contradicts the connectedness of $T_{\psi}(K)$.

Using the function $H_{\psi}^{*}$, we have also the following characterization of the edges of $T_{\psi}$.

Lemma 3.14. Let $e \in \Upsilon^{(1)}$. Then $e \in T_{\psi}(K)$ if and only if there exists $\xi=$ $\{\alpha, \beta\} \in \Upsilon_{\psi}^{(2)}(K)$, and $\gamma \in \Upsilon_{\psi}^{(3)}\left(H_{\psi}^{*}(\xi, K)\right)$, such that $e=\gamma \cap \xi$.

Proof. This is a direct consequence of the definition.

This lemma leads to the following property of Markoff maps in $\left(\boldsymbol{\Psi}_{\boldsymbol{\omega}}\right)_{Q}$.

Lemma 3.15. Let $\psi \in\left(\boldsymbol{\Psi}_{\boldsymbol{\omega}}\right)_{Q}$; then the tree $T_{\psi}(2+M)$ is a finite attracting subtree.

Proof. Suppose $\psi \in\left(\mathbf{\Psi}_{\boldsymbol{\omega}}\right)_{Q}$; then the set $\Upsilon_{\psi}^{(2)}(2+M)$ is finite, and for each element $\xi \in \Upsilon_{\psi}^{(2)}(2+M)$, we have $\psi(\xi) \notin[-2,2]$ and $\sigma(\xi) \neq 0$. Hence, the function $H_{\psi}(2+M, \xi)$ is finite, which means that the subarc $J_{\psi}(2+M, \xi)$ is finite. So the subtree $T_{\psi}(2+M)$ is a finite union of finite subarcs. 


\section{Fibonacci growth and proof of Theorem A}

In this section we define the notion of Fibonacci growth for a function defined on $\Upsilon^{(3)} \cup \Upsilon^{(2)}$, and prove that a map satisfying the (BQ)-conditions has Fibonacci growth. Note that this is a strengthening of the attracting tree property of [19], defined to show that the set $\left(\boldsymbol{\Psi}_{\boldsymbol{\omega}}\right)_{Q}$ is open. We will then use this to prove that the set $\left(\boldsymbol{\Psi}_{\boldsymbol{\omega}}\right)_{Q}$ of Markoff maps satisfying the (BQ)-conditions is an open domain of discontinuity for the mapping class group action, and we will also give different characterizations for the corresponding representations in $\mathfrak{X}_{Q}(N)$.

\subsection{Fibonacci growth.}

4.1.1. Fibonacci functions. We need to introduce the following notation. Given an oriented edge $\vec{e}$, we define the set $\Upsilon^{(k), 0}(\vec{e})$, where $k=2,3$, as the subset of $\Upsilon^{(k)}$ given by the three $k$-cells (either regions or faces) containing $\vec{e}$. Removing $\vec{e}$ from $\Upsilon^{(1)}$ leaves two disjoint subtrees $\Upsilon^{ \pm}$. Let $\Upsilon^{+}$be the one containing the head of $\vec{e}$. We define $\Upsilon^{(k),-}(\vec{e})$ to be the subset of $\Upsilon^{(k)}$ given by the $k$-cells whose boundary edges lie in $\Upsilon^{-}$, and similarly for $\Upsilon^{(k),+}(\vec{e})$. We have the following decomposition:

$$
\Upsilon^{(k)}=\Upsilon^{(k),-}(\vec{e}) \cup \Upsilon^{(k), 0}(\vec{e}) \cup \Upsilon^{(k),+}(\vec{e}) .
$$

Note that $\Upsilon^{(k),+}(\vec{e})=\Upsilon^{(k),-}(-\vec{e})$, where $-\vec{e}$ is the same edge as $\vec{e}$, pointing in the opposite direction. Let $\Upsilon^{(k), 0 \pm}(\vec{e})=\Upsilon^{(k), \pm}(\vec{e}) \cup \Upsilon^{(k), 0}(\vec{e})$.

Recall that we put the standard metric on the 1 -skeleton $\Upsilon^{(1)}$ of the complex $\Upsilon$, where every edge has length 1 . If $v$ is a vertex and $X \in \Upsilon^{(2)} \cup \Upsilon^{(3)}$, the distance $d_{v}(X)$ is given by the minimal distance in $\Upsilon^{(1)}$ between $v$ and a vertex in $X$. Given an edge $e$, we also need to define the distance $d_{e}(X)$. If $X \in \Upsilon^{(k), 0-}(\vec{e})$, then we say that $d_{e}(X)=d_{v}(X)$, where $v$ is the head of $\vec{e}$, while, if $X \in \Upsilon^{(k), 0-}(-\vec{e})$, we say that $d_{e}(X)=d_{v}(X)$, where $v$ is the head of $-\vec{e}$.

There are two types of Fibonacci functions that we will use: one defined over the set of regions $\Upsilon^{(3)}$, and one defined over the set of faces $\Upsilon^{(2)}$. This will allow us to give the notion of "Fibonacci growth". In Proposition 4.5 we will see that the two notions are related, and that they express the word length of the elements that they represent.

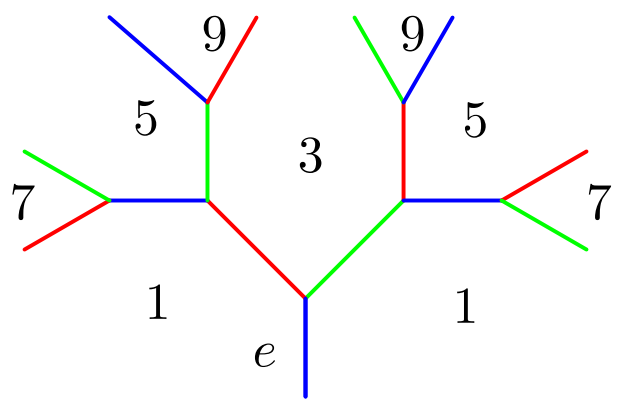

Figure 6. The Fibonacci function $F_{e}: \Upsilon^{(3)} \longrightarrow \mathbb{Z}_{\geq 0}$. At each vertex there is an additional edge, not shown in the picture. 
Fix an edge $e$ and define the function

$$
F_{e}: \Upsilon^{(3)} \longrightarrow \mathbb{Z}_{\geq 0}
$$

as follows. We set $F_{e}(\alpha)=1$ on the three regions around $e$ (on $\Upsilon^{(3), 0}(\vec{e})$ ), and for every "new" region $\alpha \in \Upsilon^{(3)}$ we set $F_{e}(\alpha)$ as the sum of the (already assigned) values of the other three regions meeting $\alpha$ at the same vertex. More formally, we define $F_{e}$ as follows:

$$
\begin{aligned}
& F_{e}(\alpha) \\
& = \begin{cases}1 & \text { if } d_{e}(\alpha)=0, \\
F_{e}(\beta)+F_{e}(\gamma)+F_{e}(\delta) & \text { if }\{\alpha, \beta, \gamma, \delta\} \in \Upsilon^{(0)} \text { and } d_{e}(\beta), d_{e}(\gamma), d_{e}(\delta)<d_{e}(\alpha) .\end{cases}
\end{aligned}
$$

This notion is due to Bowditch 2]. In Figure 6, you can see the value of $F_{e}$ on the region given by the edges in the picture, and an additional edge perpendicular to the face (and coming out of it).

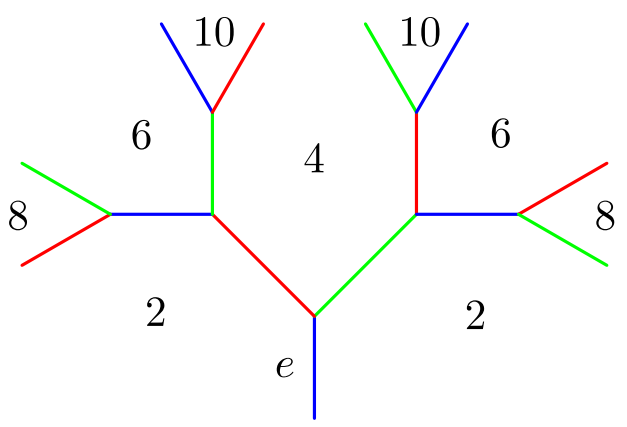

Figure 7. The Fibonacci function $F_{e}: \Upsilon^{(2)} \longrightarrow \mathbb{Z}_{\geq 0}$. (Note that at every vertex there is a fourth edge not drawn.)

If we consider the faces, we define

$$
F_{e}: \Upsilon^{(2)} \longrightarrow \mathbb{Z}_{\geq 0}
$$

to be 2 on the three faces around $e$, and then we define $F_{e}$ on the remaining faces by assigning to every "new" face $(\alpha, \beta) \in \Upsilon^{(2)}$ the sum of the (already assigned) values of the other two faces meeting $(\alpha, \beta)$ at the same vertex, and having shorter distance from $e$. More formally, we define $F_{e}$ as follows:

$$
F_{e}(\xi)= \begin{cases}2 & \text { if } d_{e}(\xi)=0 \\ F_{e}\left(\xi^{\prime}\right)+F_{e}\left(\xi^{\prime \prime}\right) & \text { if } d_{e}\left(\xi^{\prime}\right), d_{e}\left(\xi^{\prime \prime}\right)<d_{e}(\xi), \xi \cap \xi^{\prime} \in \Upsilon^{(1)}, \xi \cap \xi^{\prime \prime} \in \Upsilon^{(1)} .\end{cases}
$$

This notion is a modification of the notion of Fibonacci function given by $\mathrm{Hu}-$ Tan-Zhang [13, who define the Fibonacci function $F_{v}$ with respect to a vertex $v$. Recall also the correspondence between faces and bi-colored geodesics, underlined in Remark 2.5. In Figure 7 we only draw the faces in the boundary of a region, but in Figure 8 you can see a more complete picture.

The following lemma can be easily proved by induction. Its corollary shows that the concept of upper and lower Fibonacci bound is independent of the edge $e$ used. 


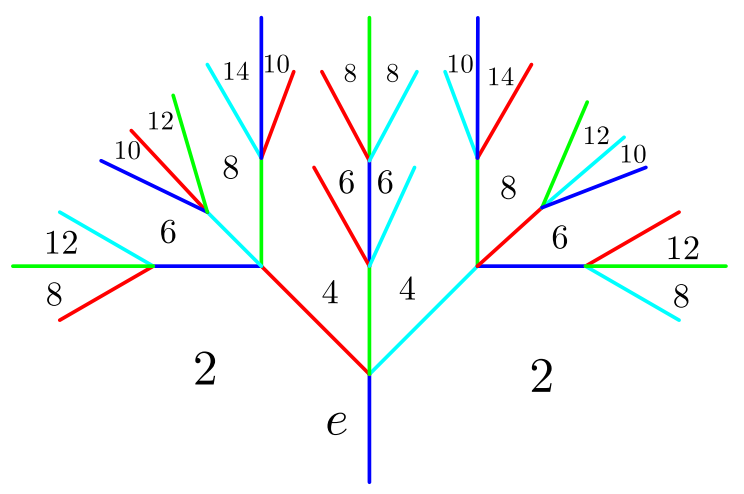

Figure 8. The Fibonacci function $F_{e}$ on (some of) the faces (or bi-colored geodesics).

Lemma 4.1 (Lemma 2.1.1 2]; Lemma 24-25 14]). Let $e$ be an edge that is the intersection of the three regions $\alpha_{1}, \alpha_{2}, \alpha_{3}$ and let $f: \Upsilon^{(3)} \rightarrow[0, \infty)$ be a function. Let $M=\max \left\{f\left(\alpha_{1}\right), f\left(\alpha_{2}\right), f\left(\alpha_{3}\right)\right\}, m=\min \left\{f\left(\alpha_{1}\right), f\left(\alpha_{2}\right), f\left(\alpha_{3}\right)\right\}$. Let $\alpha, \beta, \gamma, \delta$ be four regions that meet at the same vertex and such that $\delta$ is strictly farther from $e$ than $\alpha, \beta, \gamma$. Then:

(1) If there exists $c>0$ such that for all such $\alpha, \beta, \gamma, \delta$ one has $f(\delta) \leq f(\alpha)+$ $f(\beta)+f(\gamma)+2 c$, then for all but finitely many $X \in \Upsilon^{(3)}$ one has $f(X) \leq$ $(M+c) F_{e}(X)-c$.

(2) If there exists $0 \leq c<m$ such that for all such $\alpha, \beta, \gamma$, $\delta$ one has $f(\delta) \geq$ $f(\alpha)+f(\beta)+f(\gamma)-2 c$, then for all but finitely many $X \in \Upsilon^{(3)}$ one has $f(X) \geq(m-c) F_{e}(X)+c$.

Corollary 4.2 (Corollary 2.1.2 in [2]). Suppose $f: \Upsilon^{(3)} \rightarrow[0, \infty)$ satisfies an inequality of the form $f(\delta) \leq f(\alpha)+f(\beta)+f(\gamma)+c$ (resp., $f(\delta) \geq f(\alpha)+f(\beta)+f(\gamma)+$ $c)$ for some fixed constant $c$, whenever $\alpha, \beta, \gamma, \delta \in \Upsilon^{(3)}$ meet at a vertex. Then for any given edge $e \in \Upsilon^{(1)}$, there is a constant $K>0$, such that $f(X) \leq K F_{e}(X)$ (resp., $f(X) \geq K F_{e}(X)$ ) for all $X \in \Upsilon^{(3)}$.

Note that the same result holds for $\Upsilon^{(2)}$; see Proposition 4.5 .

4.1.2. Fibonacci growth. We can now define when a function $f: \Upsilon^{(3)} \longrightarrow \mathbb{R}$, or $g: \Upsilon^{(2)} \longrightarrow \mathbb{R}$, has an upper or lower Fibonacci bound, or Fibonacci growth. Notice that this definition says something about the asymptotic growth of the function, and hence it is independent of the edge $e$ used in the definition of the respective Fibonacci function.

Definition 4.3. Suppose $f: \Upsilon^{\prime} \subset \Upsilon^{(3)} \longrightarrow \mathbb{R}$ or $g: \Upsilon^{\prime} \subset \Upsilon^{(2)} \longrightarrow \mathbb{R}$. We say that:

- $f$ (resp., $g$ ) has an upper Fibonacci bound on $\Upsilon^{\prime}$ if there is some constant $\kappa>0$ such that $f(X) \leq \kappa F_{e}(X)$ (resp., $g(X) \leq \kappa F_{e}(X)$ ) for all but finitely many $X \in \Upsilon^{\prime}$;

- $f$ (resp., $g$ ) has a lower Fibonacci bound on $\Upsilon^{\prime}$ if there is some constant $\kappa>0$ such that $f(X) \geq \kappa F_{e}(X)$ (resp., $g(X) \geq \kappa F_{e}(X)$ ) for all but finitely many $X \in \Upsilon^{\prime}$; 
- $f$ (resp., g) has Fibonacci growth on $\Upsilon^{\prime}$ if it has both upper and lower Fibonacci bounds on $\Upsilon^{\prime}$;

- $f$ (resp., $g$ ) has Fibonacci growth if it has Fibonacci growth on all of $\Upsilon^{(3)}$ (resp., $\left.\Upsilon^{(2)}\right)$.

Remark 4.4. Note that, equivalently, we can say that:

- $f$ (resp., $g$ ) has an upper Fibonacci bound on $\Upsilon^{\prime}$ if there are some constants $\kappa, C>0$ such that $f(X) \leq \kappa F_{e}(X)+C$ (resp., $\left.g(X) \leq \kappa F_{e}(X)+C\right)$ for all $X \in \Upsilon^{\prime}$;

- $f$ (resp., $g$ ) has a lower Fibonacci bound on $\Upsilon^{\prime}$ if there are some constants $\kappa, C>0$ such that $f(X) \geq \kappa F_{e}(X)-C$ (resp., $\left.g(X) \geq \kappa F_{e}(X)-C\right)$ for all $X \in \Upsilon^{\prime}$.

The function that we will consider is the function $\log ^{+}|\psi|: \Upsilon^{(2)} \cup \Upsilon^{(3)} \longrightarrow \mathbb{R}$ defined by $\log ^{+}|\psi|:=\max \{\log |\psi|, 0\}$, where $\psi \in \mathbf{\Psi}_{\boldsymbol{\omega}}$. For any $\boldsymbol{\omega}$-Markoff map $\psi$, we will prove that the function $\log ^{+}|\psi|$ will have an upper Fibonacci bound.

On the other hand, we will need to restrict to maps $\psi$ in the Bowditch domain $\left(\boldsymbol{\Psi}_{\boldsymbol{\omega}}\right)_{Q}$ to prove that $\log ^{+}|\psi|$ has Fibonacci growth on $\Upsilon^{(3)}$. The following result will then show that $\log ^{+}|\psi|$ has Fibonacci growth on $\Upsilon^{(2)}$ as well.

Proposition 4.5. Let $\psi \in\left(\boldsymbol{\Psi}_{\boldsymbol{\omega}}\right)_{Q}$. The function $\log ^{+}|\psi|$ has Fibonacci growth on $\Upsilon^{(3)}$ if and only if $\log ^{+}|\psi|$ has Fibonacci growth on $\Upsilon^{(2)}$.

Proof. First, note that given a face $\xi=\{\alpha, \beta\} \in \Upsilon^{(2)}$, we have that

$$
|\psi(\alpha)| \cdot|\psi(\beta)|-M \leq|\psi(\xi)| \leq|\psi(\alpha)| \cdot|\psi(\beta)|+M .
$$

Since $\psi \in\left(\boldsymbol{\Psi}_{\boldsymbol{\omega}}\right)_{Q}$, we know that the set $\Upsilon_{\psi}^{(2)}(2+M)$ is finite, and so there is just a finite number of faces such that $|\psi(\xi)| \leq(M+2)^{2}+M$. We can then suppose $|a b-x|>(M+2)^{2}+M$, and so $|a b|>(M+2)^{2}$. In that case we have

$$
\frac{M+1}{M+2}|a b| \leq|a b-x| \leq \frac{M+3}{M+2}|a b|,
$$

because

$$
|a b|-M=\frac{M+1}{M+2}|a b|+\frac{1}{M+2}|a b|-M>\frac{M+1}{M+2}|a b|+(M+2)-M>\frac{M+1}{M+2}|a b|,
$$

and similarly

$$
|a b|+M=\frac{M+3}{M+2}|a b|-\frac{1}{M+2}|a b|-M<\frac{M+3}{M+2}|a b|-(M+2)+M<\frac{M+3}{M+2}|a b| .
$$

Hence we can see that

$$
\log ^{+}|\psi(\alpha)|+\log ^{+}|\psi(\beta)|-K \leq \log ^{+}|\psi(\xi)| \leq \log ^{+}|\psi(\alpha)|+\log ^{+}|\psi(\beta)|+K^{\prime},
$$

and so, using Proposition 4.6, we can conclude that if the function $\log ^{+}|\psi|$ has Fibonacci growth on $\Upsilon^{(2)}$, then it has Fibonacci growth on $\Upsilon^{(3)}$.

Conversely, we can see that

$$
\frac{M+2}{M+3}|a b-x| \leq|a b| \leq \frac{M+2}{M+1}|a b-x| .
$$

Similarly, we have

$$
\frac{M+2}{M+3}|a c-z| \leq|a c| \leq \frac{M+2}{M+1}|a c-z|,
$$


and

$$
\frac{M+2}{M+3}|b c-y| \leq|b c| \leq \frac{M+2}{M+1}|b c-y| .
$$

So, using Proposition 4.6, and adding equations (10) and (11), and subtracting (12), we can conclude that if $\log ^{+}|\psi|$ has Fibonacci growth on $\Upsilon^{(3)}$, then it has Fibonacci growth on $\Upsilon^{(2)}$.

Another important meaning of these Fibonacci functions is explained by the following result, relating $F_{e}$ to the word length of the elements of $\mathcal{S} \subset \Gamma / \sim$. (See Section 2.2 for the definition of $\Gamma / \sim$.)

Proposition 4.6. Suppose $\alpha, \beta$, and $\gamma$ form a set of free generators for $\Gamma$ corresponding to regions in $\Upsilon^{(3)}$ with the same name. Let $e$ be the edge $e=\alpha \cap \beta \cap \gamma$. If $\omega_{X} \in \mathcal{S}$ corresponds to $X \in \Upsilon^{(k)}$, where $k=2$ or $k=3$, then $\mathrm{W}\left(\omega_{X}\right)=F_{e}(X)$.

In addition, for any face $\xi_{\alpha, \beta}=\alpha \cap \beta$, where $\alpha, \beta \in \Upsilon^{(3)}$, we have $F_{e}\left(\xi_{\alpha, \beta}\right)=$ $F_{e}(\alpha)+F_{e}(\beta)$.

Proof. We will prove, inductively on the integer $d$, the following claim: If four regions $\alpha_{0}, \alpha_{1}, \alpha_{2}, \alpha_{3} \in \Upsilon^{(3)}$ meet at a vertex which is at distance $d$ from the starting edge $e$, such that $\alpha_{3}$ is the furthermost region from $e$, then there exists a standard generating set $\left(u_{0}, u_{1}, u_{2}\right)$, composed of cyclically reduced words representatives of $\alpha_{0}, \alpha_{1}, \alpha_{2}$ such that $u_{3}=u_{0} u_{1} u_{2}$ is a cyclically reduced representative of $\alpha_{3}$ and such that $u_{0} u_{1}^{-1}, u_{1} u_{2}^{-1}, u_{2} u_{0}^{-1}$ are cyclically reduced representatives of the faces $\xi_{\alpha_{1}, \alpha_{2}}, \xi_{\alpha_{2}, \alpha_{3}}$ and $\xi_{\alpha_{3}, \alpha_{1}}$.

Recall that cyclically reduced representatives of a simple closed curve are words in the free group mutually related by cyclic permutation and/or inversion. When the representatives are cyclically reduced, the word lengths satisfy the equalities $\mathrm{W}\left(\alpha_{4}\right)=\mathrm{W}\left(\alpha_{1}\right)+\mathrm{W}\left(\alpha_{2}\right)+\mathrm{W}\left(\alpha_{3}\right)$ and $\mathrm{W}\left(\xi_{\alpha_{i}, \alpha_{j}}\right)=\mathrm{W}\left(\alpha_{i}\right)+\mathrm{W}\left(\alpha_{j}\right)$, which will prove the proposition.

Let's first prove the claim for vertices at distance 0 of the edge $e$. Let $\alpha, \beta$, and $\gamma$ be a set of standard free generators for $\Gamma$, and let $e=\left(\alpha, \beta, \gamma ; \delta, \delta^{\prime}\right)$. The regions $\alpha, \beta, \gamma \in \mathcal{S}_{1}$ are represented, respectively, by the generators with the same names. Without loss of generality, we can suppose that $\delta$ and $\delta^{\prime}$ are represented by $\alpha \beta \gamma$ and $\gamma \beta \alpha$, respectively, and that the face $\xi_{\alpha, \beta}=\alpha \cap \beta \in \mathcal{S}_{2}$ is represented by $\alpha \beta^{-1}$. So the claim is true for the vertices at distance 0 of the edge $e$.

Assume that we have a vertex $v=\left(\alpha_{0}, \alpha_{1}, \alpha_{2}, \alpha_{3}\right)$ at distance $d$ from the starting edge, with $\alpha_{3}$ the furthermost region. And let $\left(u_{0}, u_{1}, u_{2}\right)$ be a standard generating set satisfying the induction hypothesis. We consider the adjacent vertex at distance $d+1$ that is opposite to $\alpha_{0}$ across the edge $\left(\alpha_{1}, \alpha_{2}, \alpha_{3} ; \alpha_{0}, \alpha_{4}\right)$. This new vertex is surrounded by the regions $\left(\alpha_{1}, \alpha_{2}, \alpha_{3}, \alpha_{4}\right)$. Using Remark 2.3. we know that the triple $\left(u_{2} u_{0} u_{1}, u_{2}^{-1}, u_{1}^{-1}\right)$ is a standard generating set, and hence we can infer that the element $u_{4}=u_{2} u_{0} u_{1} u_{2}^{-1} u_{1}^{-1}$ is a 1-sided simple closed curve that intersects $\alpha_{1}, \alpha_{2}$, and $\alpha_{3}$ once, and is different from $\alpha_{0}$, which means that $u_{4}$ is a representative of the region $\alpha_{4}$.

The induction hypothesis states that the three words $u_{0} u_{1} u_{2}, u_{1} u_{2}^{-1}, u_{2}^{-1} u_{1}^{-1}$ are reduced. So the new element $u_{4}$ is also a cyclically reduced word, as any two (cyclically) consecutive terms among the five factors $u_{2}, u_{0}, u_{1}, u_{2}^{-1}, u_{1}^{-1}$ form a reduced word. 
The three words $u_{2} u_{0} u_{1} u_{2}, u_{2}^{-1} u_{1}$, and $u_{1}^{-2} u_{0}^{-1} u_{2}^{-1}$, which are representatives of the three faces $\xi_{\alpha_{1}, \alpha_{2}}, \xi_{\alpha_{2}, \alpha_{3}}$, and $\xi_{\alpha_{3}, \alpha_{1}}$, are also cyclically reduced using the same argument. This proves the hypothesis for this vertex at distance $d+1$.

A similar reasoning can be used for the other two vertices at distance $d+1$ that are adjacent to $v$, using the other standard generating triples mentioned in Remark 2.2. This proves the induction step and ends the proof of the proposition.

4.1.3. Upper Fibonacci bound. In this section we will show that, for any $\boldsymbol{\omega}$-representation $\psi$, the function $\log ^{+}|\psi|$ has an upper Fibonacci bound on $\Upsilon^{(3)}$ (and so on $\Upsilon^{(2)}$, by Proposition 4.5).

Lemma 4.7. If $\psi \in \mathbf{\Psi}_{\boldsymbol{\omega}}$, then $\log ^{+}|\psi|$ has an upper Fibonacci bound on $\Upsilon^{(3)}$.

Proof. We will follow ideas from [24]. Let $\alpha, \beta, \gamma, \delta \in \Upsilon^{(3)}$ be four regions that meet at a vertex, and let $a=\psi(\alpha), b=\psi(\beta)$, etc. We will prove that

$$
\log ^{+}|d| \leq \log ^{+}|a|+\log ^{+}|b|+\log ^{+}|c|+C,
$$

where $C$ is a constant depending only on $|x|,|y|$, and $|z|$, and then conclude using Corollary 4.2 .

If $|d| \leq 2 \max \{|a|,|b|,|c|\}$, then (13) holds already. So we suppose

$$
|d|>2 \max \{|a|,|b|,|c|\} .
$$

Then, since

$-a b c d+x(a b+c d)+y(b c+a d)+z(a c+b d)+4-x^{2}-y^{2}-z^{2}-x y z=a^{2}+b^{2}+c^{2}+d^{2}$, we have:

$$
\begin{aligned}
& |a b c d|+|x||a b|+|x||c d|+|y||b c|+|y||a d|+|z||a c|+|z||b d|+\left|4-x^{2}-y^{2}-z^{2}-x y z\right| \\
& \geq|d|^{2}-|a|^{2}-|b|^{2}-|c|^{2} \\
& =|d|^{2} / 4+\left(|d|^{2} / 4-|a|^{2}\right)+\left(|d|^{2} / 4-|b|^{2}\right)+\left(|d|^{2} / 4-|c|^{2}\right) \\
& \geq|d|^{2} / 4 .
\end{aligned}
$$

Hence $|d|^{2} \leq 32 m$, where

$m=\max \left\{|a b c d|,|x||a b|,|y||b c|,|z||a c|,|x||c d|,|y||a d|,|z||b d|,\left|4-x^{2}-y^{2}-z^{2}-x y z\right|\right\}$.

So, according to the value of $m$, we have, respectively:

(1) $|d|^{2} \leq 32|a b c d|$, so $|d| \leq 32|a b c|$ and

$$
\log ^{+}|d| \leq \log ^{+}|a|+\log ^{+}|b|+\log ^{+}|c|+\log ^{+}(32)
$$

(2) $|d|^{2} \leq 32|x||a b|$, so $|d| \leq|d|^{2} \leq 32|x||a b|$ and $\log ^{+}|d| \leq \log ^{+}|a|+\log ^{+}|b|$ $+\log ^{+}(32)+\log ^{+}|x|$ so

$$
\log ^{+}|d| \leq \log ^{+}|a|+\log ^{+}|b|+\log ^{+}|c|+\log ^{+}(32)+\log ^{+}|x| ;
$$

(3) $|d|^{2} \leq 32|x||c d|$, so $|d| \leq 32|x||c|$ and $\log ^{+}|d| \leq \log ^{+}|c|+\log ^{+}|x|+\log ^{+}(32)$, hence

$$
\log ^{+}|d| \leq \log ^{+}|a|+\log ^{+}|b|+\log ^{+}|c|+\log ^{+}(32)+\log ^{+}|x|
$$

(4) $|d|^{2} \leq 32\left|4-x^{2}-y^{2}-z^{2}-x y z\right|$, so $|d| \leq|d|^{2} \leq 32\left|4-x^{2}-y^{2}-z^{2}-x y z\right|$ and $\log ^{+}(|d|) \leq \log ^{+}(32)+\log ^{+}\left|4-x^{2}-y^{2}-z^{2}-x y z\right|$, hence

$\log ^{+}(|d|) \leq \log ^{+}|a|+\log ^{+}|b|+\log ^{+}|c|+\log ^{+}(32)+\log ^{+}\left|4-x^{2}-y^{2}-z^{2}-x y z\right|$; 
or a similar inequality. Note that the situation where $|d|<1$ is irrelevant here because we are taking $\log ^{+}$and not log. We have only a finite number of cases to consider and in each case we have an inequality of the form $\log ^{+}|d| \leq \log ^{+}|a|+\log ^{+}|b|+$ $\log ^{+}|c|+C_{i}$ with $C_{i}$ a constant depending only on $|x|,|y|$, and $|z|$. So we can take $C$ as the max of the constants $C_{i}$ appearing in each case, which proves equation (13).

4.1.4. Lower Fibonacci bound. In this section we will show that, for any $\boldsymbol{\omega}$-representation $\psi$ in the Bowditch set, the function $\log ^{+}|\psi|$ will have a lower Fibonacci bound (and so Fibonacci growth) on $\Upsilon^{(3)}$. We will then use that to prove that $\log ^{+}|\psi|$ has Fibonacci growth on $\Upsilon^{(2)}$.

Theorem 4.8. If $\psi \in\left(\mathbf{\Psi}_{\boldsymbol{\omega}}\right)_{Q}$, then $\log ^{+}|\psi|$ has lower Fibonacci growth on $\Upsilon^{(3)}$.

Proof. The proof uses many arguments that are already in the proofs of Lemma 4.9 and 4.10 in 19 and the structure of the proof of Proposition 3.11 in 13 .

From Lemma 3.15, we know that, if $\psi \in\left(\mathbf{\Psi}_{\boldsymbol{\omega}}\right)_{Q}$, then there is a finite $\psi$ attracting subtree $T_{\psi}=T_{\psi}(2+M)$, where $M=\max \{|x|,|y|,|z|\}$. Remember that the circular neighborhood $C\left(T_{\psi}\right)$ is the set of (directed) edges surrounding the finite subtree $T_{\psi}$, as defined in Proposition 3.13. So the complement of $\Upsilon_{\psi}^{(3)}(2+M)$ consists of $\bigcup_{\vec{e} \in C\left(T_{\psi}\right)} \Upsilon^{(3), 0-}(\vec{e})$. So it suffices to prove that for each edge $\vec{e}$ in $C\left(T_{\psi}\right)$, $\log ^{+}|\psi|$ has lower Fibonacci growth on $\Upsilon^{(3), 0-}(\vec{e})$.

We distinguish two cases.

Case 1. $\Upsilon^{(2), 0-}(\vec{e}) \cap \Upsilon_{\psi}^{(2)}(2+M)=\emptyset$.

This implies that $\Upsilon^{(3), 0-}(\vec{e}) \cap \Upsilon_{\psi}^{(3)}(2+M)$ consists of at most one region. Otherwise, there would be two regions in $\Upsilon^{(3), 0-}(\vec{e}) \cap \Upsilon_{\psi}^{(3)}(2+M)$ and by the connectivity of $\Upsilon_{\psi}^{(3)}(2+M)$ we could assume that these two regions are adjacent. Then, Lemma 3.1 implies that these two regions would intersect in a face in $\Upsilon_{\psi}^{(2)}(2+M)$, contradicting the assumption.

Now let $\alpha \in \Upsilon^{(3),-}(\vec{e}) \cap \Upsilon_{\psi}^{(3)}(2+M)$, and let $\beta, \gamma, \delta, \alpha^{\prime} \in \Upsilon^{(3),-}(\vec{e})$ having shorter distance from $e$, and such that $(\alpha, \beta, \gamma, \delta) \in \Upsilon^{(0)}$ and $\left(\alpha^{\prime}, \beta, \gamma, \delta\right) \in \Upsilon^{(0)}$. Without loss of generality, we may assume that $|b| \geq|c| \geq|d|$. As there are no faces in $\Upsilon_{\psi}^{(2)}(2+M)$, we have that $|c d|>(2+M)^{2}$.

Hence, we obtain:

$$
\begin{aligned}
2|a| & \geq\left|a+a^{\prime}\right|=|b c d-x b-z c-y d| \\
& \geq|b c d|-|x||b|-|z||c|-|y||d| \\
& \geq|b c d|-M(|b|+|c|+|d|) \\
& \geq \frac{1}{2}|b c d|+\left(\frac{1}{2}|b c d|-M(|b|+|c|+|d|)\right) \\
& \geq \frac{1}{2}|b c d|+|b|\left(\frac{1}{2}(2+M)^{2}-3 M\right) \\
& \geq \frac{1}{2}|b c d| .
\end{aligned}
$$

This gives:

$$
\log ^{+}|a| \geq \log ^{+}|b|+\log ^{+}|c|+\log ^{+}|d|-2 \log 2 .
$$


Now, using Lemma 4.1 (2), we can conclude that there is a Fibonnacci lower bound on $\Upsilon^{(3), 0-}(\vec{e})$ with $\kappa=m-c, m=\min \left\{\log ^{+}|b|, \log ^{+}|c|, \log ^{+}|d|\right\}=\log ^{+}|d|$ and $C=c=\log 2$.

Case 2. $\Upsilon^{(2), 0-}(\vec{e}) \cap \Upsilon_{\psi}^{(2)}(2+M) \neq \emptyset$.

First, we note that $\Upsilon^{(2), 0-}(\vec{e}) \cap \Upsilon_{\psi}^{(2)}(2+M)=\{\xi\}$, with $\xi=\{\alpha, \beta\}$ a face in $\Upsilon^{(2), 0}(\vec{e})$. The fact that we have only one face follows from the definition of $T_{\psi}$ : if the intersection consists of more than two faces, then by edge-connectedness of $\Upsilon_{\psi}^{(2)}(2+M)$, we could assume that these two faces share an edge. On the other hand, by the construction of the tree $T_{\psi}$, if two adjacent faces are in $\Upsilon_{\psi}^{(2)}(2+M)$, then the edge corresponding to the intersection of these two faces is in $T_{\psi}$. This gives a contradiction as $T_{\psi}$ is connected and $\vec{e} \notin T_{\psi}$. A similar argument also shows that if $\Upsilon^{(2), 0}(\vec{e}) \cap \Upsilon_{\psi}^{(2)}(2+M)=\emptyset$, then $\Upsilon^{(2),-}(\vec{e}) \cap \Upsilon_{\psi}^{(2)}(2+M)=\emptyset$, so $\xi$ should be in $\Upsilon^{(2), 0}(\vec{e}) \cap \Upsilon_{\psi}^{(2)}(2+M)$.

Now, let $\left(e_{n}\right)_{n=0}^{\infty}$ be the sequence of directed edges lying in the boundary of the face $\xi$ and in $\Upsilon^{0-}(\vec{e})$, such that $e_{0}=e$. Let $\gamma_{n}$ for $n \geq 0$, be the regions such that $\gamma_{n} \cap \xi=e_{n}$. We denote each vertex by $v_{n}$ so that $v=v_{0}$.

At each vertex $v_{n}$ there are two edges $\overrightarrow{\eta_{n}}$ and $\overrightarrow{\varepsilon_{n}}$ incident to this vertex that do not belong to $\xi$. We can write that:

$$
\Upsilon^{(3), 0-}(\vec{e})=\left(\bigcup_{n \geq 1}\left\{\gamma_{n}\right\}\right) \cup\left(\bigcup_{n \geq 1} \Upsilon^{(3), 0-}\left(\overrightarrow{\eta_{n}}\right) \cup \Upsilon^{(3), 0-}\left(\overrightarrow{\varepsilon_{n}}\right)\right)
$$

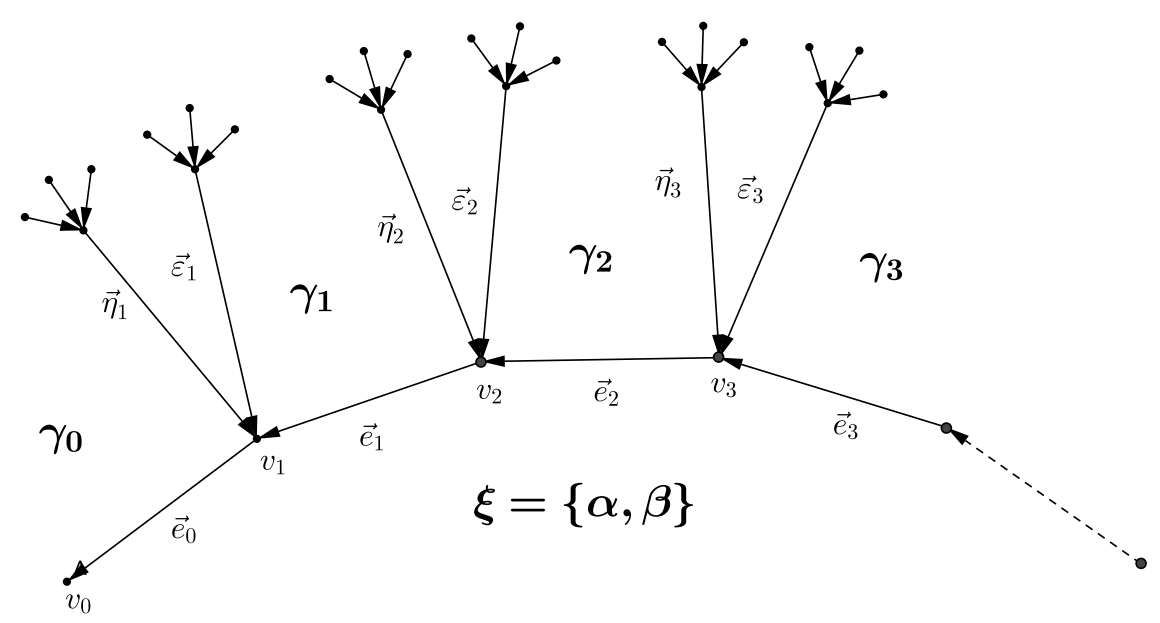

FiguRE 9 . The labelling of the vertices in the boundary of a face $\xi$ intersecting $\Upsilon^{-}$. 
Using the previous case, we have the Fibonacci lower bound on $\Upsilon^{(3), 0-}\left(\overrightarrow{\eta_{n}}\right)$ and $\Upsilon^{(3), 0-}\left(\overrightarrow{\varepsilon_{n}}\right)$ for each $n$. So there exist constants $\kappa_{n}$ and $C_{n}$ (resp., $\kappa_{n}^{\prime}$ and $C_{n}^{\prime}$ ) given at the end of the previous case, such that for all $X \in \Upsilon^{(3), 0-}\left(\overrightarrow{\eta_{n}}\right)$ we have $\log ^{+}|\psi(X)| \geq \kappa_{n} F_{\eta_{n}}(X)-C_{n}$ (resp., for all $X \in \Upsilon^{(3), 0-}\left(\overrightarrow{\epsilon_{n}}\right)$ we have $\log ^{+}|\psi(X)| \geq$ $\left.\kappa_{n}^{\prime} F_{\epsilon_{n}}(X)-C_{n}^{\prime}\right)$. Note that the constants $C_{n}=C_{n}^{\prime}=\log 2$ do not depend on $n$, while the constants $\kappa_{n}$ and $\kappa_{n}^{\prime}$ do. On the other hand, the minima of the values of $\psi$ on the regions around the edges $\eta_{n}$ and $\varepsilon_{n}$ used in the formula increase with $n$, so the sequences $\kappa_{n}$ and $\kappa_{n}^{\prime}$ are increasing. Hence for all $n$ we can use $\min \left(\kappa_{1}, \kappa_{1}^{\prime}\right)$ for both $\Upsilon^{(3), 0-}\left(\overrightarrow{\eta_{n}}\right)$ and $\Upsilon^{(3), 0-}\left(\overrightarrow{\epsilon_{n}}\right)$ to obtain uniform constants in the Fibonnacci lower bound.

The last ingredient is to prove the Fibonacci bound on regions $\gamma_{n}$. This is a consequence of Lemma 3.9 that proves that $\left|c_{n}\right|$ grows exponentially. Hence, there exists $\kappa>0$ such that $\log ^{+}\left|\psi\left(\gamma_{n}\right)\right| \geq \kappa n \geq \kappa F_{e}\left(\gamma_{n}\right)$. Combining both arguments, we get that for all but finitely many $\delta \in \Upsilon^{(3), 0-}\left(\eta_{n}\right)$, we have

$$
\log ^{+}|\psi(\delta)|>\kappa_{1} n F_{\eta_{n}}(\delta) \geq \kappa_{1} F_{e}(\delta),
$$

and similarly for all but finitely many $\delta \in \Upsilon^{(3), 0-}\left(\varepsilon_{n}\right)$, we have

$$
\log ^{+}|\psi(\delta)|>\kappa_{1}^{\prime} n F_{\varepsilon}(\delta) \geq \kappa_{1}^{\prime} F_{e}(\delta) .
$$

This proves Fibonnacci growth on $\Upsilon^{(3),-}(\vec{e})$.

Using Lemma 4.7, Theorem 4.8, and Proposition 4.5, we have now the following result.

Corollary 4.9. If $\psi \in\left(\boldsymbol{\Psi}_{\boldsymbol{\omega}}\right)_{Q}$, then $\log ^{+}|\psi|$ has Fibonacci growth on $\Upsilon^{(2)}$ and $\Upsilon^{(3)}$.

4.2. Characterization of BQ-set. We can now state two different characterizations for the representations in the Bowditch set, and we will use the result from the previous section to prove that the two definitions are equivalent.

First, let's see the equivalence with the definition of the Bowditch set given in the introduction and the one given in Definition 3.3. Such equivalence will be a corollary of the following proposition and Remark 2.7.

Proposition 4.10. A map $\psi$ belongs to $\left(\mathbf{\Psi}_{\boldsymbol{\omega}}\right)_{Q}$ if and only if the following two conditions are satisfied:

(BQ1) For all $\xi \in \Upsilon^{(2)}$, we have $\psi(\xi) \notin[-2,2]$.

(BQ2) For all $K>0$, the set $\Upsilon_{\psi}^{(2)}(K)$ is finite.

Proof. If $\psi \in\left(\mathbf{\Psi}_{\boldsymbol{\omega}}\right)_{Q}$, then, by Corollary 4.9, the map $\log ^{+}|\psi|$ has Fibonacci growth on $\Upsilon^{(2)}$, which means that for all $K>0$, the set $\Upsilon_{\psi}^{(2)}(K)$ is finite.

Conversely, if $\psi$ is not in $\left(\boldsymbol{\Psi}_{\boldsymbol{\omega}}\right)_{Q}$, then at least one of the three possibilities occurs:

(i) There exists $\xi \in \Upsilon^{(2)}$, such that $\psi(\xi) \in[-2,2]$.

(ii) The set $\Upsilon_{\psi}^{(2)}(2+M)$ is infinite.

(iii) There exists $\xi \in \Upsilon^{(2)}$, such that $\sigma(\xi)=0$.

Then condition (i) contradicts condition (BQ1), while condition (ii) contradicts condition (BQ2). Finally, in case (iii), the neighbors around the face $\xi$ will converge to some finite value, which means that there exists $K>0$ such that $\Upsilon_{\psi}^{(2)}(K)$ is infinite, contradicting the condition (BQ2). 
Remark 4.11. Let $\rho$ be a representation and let $\psi$ be the corresponding Markoff map. There is an equivalence between the conditions

- There exists $\xi \in \Upsilon^{(2)}$, such that $\sigma_{\psi}(\xi)=0$.

- There exists an embedded subsurface $S \subset N_{1,3}$ which is either a three-holed sphere or a two-holed projective plane and such that the restriction $\left.\rho\right|_{\pi_{1}(S)}$ is reductive.

Proof. If $\alpha$ and $\beta$ are two curves such that $\xi=\xi_{\alpha, \beta}$, then the curve $\alpha \beta^{-1}$ splits $N$ into a three-holed sphere $S^{\prime}$ and a two-holed projective plane $N^{\prime}$. The fundamental group $\pi_{1}\left(N^{\prime}\right)$ is generated by $\{\alpha, \beta\}$ and hence the restriction of $\rho$ to $\pi_{1}\left(N^{\prime}\right)$ is reductive if and only if

$$
\operatorname{tr}([\alpha, \beta])=a^{2}+b^{2}+x^{2}-a b x-2=2 .
$$

Similarly, the fundamental group $\pi_{1}\left(S^{\prime}\right)$ is generated by two elements $Y=\alpha \gamma$ and $Z=\gamma^{-1} \beta^{-1}$ which are conjugated to the boundaries of $N$ and such that $Y Z=\alpha \beta^{-1}$. (Remember the set of unoriented simple closed curves in $N$ is welldefined only as a subset of $\Gamma / \sim$; see Section 2.1 for the definition.) The restriction of $\rho$ to $\pi_{1}\left(S^{\prime}\right)$ is reductive if and only if

$$
\operatorname{tr}([Y, Z])=y^{2}+z^{2}+\psi(\xi)^{2}-y z \psi(\xi)-2=2 .
$$

As $\sigma_{\psi}(\xi)=(\operatorname{tr}([\alpha, \beta])-2)(\operatorname{tr}([Y, Z])-2)$ (see equation (8) $)$, we get the equivalence directly.

The following second correspondence is particularly interesting because it could be used to generalize the notion of Bowditch representations for general surfaces. Given $\rho$ in the character variety $\mathfrak{X}$, we can define the function $\mathrm{L}_{\rho}=\mathrm{L}(\rho(\cdot)): \mathcal{S} \longrightarrow$ $\mathbb{C}$, well-defined modulo $2 i \pi$, where $\mathcal{S}=\mathcal{S}(N)$ is the set of simple closed curves of $N=N_{1,3}$ by

$$
\operatorname{tr}(\rho(\gamma))=2 \cosh (\mathrm{L}(\rho(\gamma)) / 2)
$$

The function $\mathrm{L}_{\rho}$ is the complex translation length function and its real part corresponds to the translation length. Note that for all representations $\rho \in \mathfrak{X}_{\boldsymbol{\omega}}$, there exists $K=K(\rho)$ such that $\log |\operatorname{tr}(\rho(\gamma))| \leq K \mathrm{~W}(\gamma)$; see for example Bowditch 2, Section 4]. Recall that $\mathrm{W}$ denote the minimal cyclically reduced word length with respect to some generating set.

Proposition 4.12. The following sets are equal:

(1) the Bowditch set $\left(\mathfrak{X}_{\omega}\right)_{Q}$;

(2) $\left\{\rho \in \mathfrak{X}_{\boldsymbol{\omega}}|\exists k=k(\rho),| \mathrm{L}(\rho(\gamma)) \mid \geq k \mathrm{~W}(\gamma) \forall \gamma \in \mathcal{S}\right\}$;

(3) $\left\{\rho \in \mathfrak{X}_{\boldsymbol{\omega}}|\exists k=k(\rho),| \mathrm{L}(\rho(\gamma)) \mid \geq k \mathrm{~W}(\gamma) \forall \gamma \in \mathcal{S}_{1}\right\}$;

(4) $\left\{\rho \in \mathfrak{X}_{\boldsymbol{\omega}}|\exists k=k(\rho),| \mathrm{L}(\rho(\gamma)) \mid \geq k \mathrm{~W}(\gamma) \forall \gamma \in \mathcal{S}_{2}\right\}$.

Proof. Let $\mathfrak{S}, \mathfrak{S}_{1}, \mathfrak{S}_{2}$ be the sets defined in (2), (3), (4) of Proposition 4.12, respectively. Note that $\mathfrak{S} \subset \mathfrak{S}_{1}$ and $\mathfrak{S} \subset \mathfrak{S}_{2}$.

$\left(\mathfrak{X}_{\boldsymbol{\omega}}\right)_{Q} \subset \mathfrak{S} \subset \mathfrak{S}_{2}$ : Given $\rho \in\left(\mathfrak{X}_{\boldsymbol{\omega}}\right)_{Q}$, let $\psi \in\left(\boldsymbol{\Psi}_{\boldsymbol{\omega}}\right)_{Q}$ be the corresponding $\boldsymbol{\omega}-$ Markoff map. Proposition 4.6 tells us that, for the appropriate edge $e$, we have $\mathrm{W}(\gamma)=F_{e}(\gamma)$, if $\gamma \in \Upsilon^{(2)} \cup \Upsilon^{(3)}$. By Corollary 4.9 there is a constant $k>0$ such that $\log ^{+}|\psi(X)| \geq k F_{e}(X)$ for all but finitely many $X \in \Upsilon^{(2)} \cup \Upsilon^{(3)}$. Recall that $\psi(X)=\operatorname{tr}(X)$, so, from the inequality $\log ^{+}|\operatorname{tr}(A)| \leq|\mathrm{L}(A)|$, we can see that $|\mathrm{L}(\rho(\gamma))| \geq \log ^{+}|\psi(X)| \geq k F_{e}(X)=k \mathrm{~W}(\gamma)$. The finitely many exceptions all satisfy $|\mathrm{L}(\rho(\gamma))|>0$ so up to changing the constant $k$, we get that $|\mathrm{L}(\rho(\gamma))|>k \mathrm{~W}(\gamma)$ for all $\gamma \in \mathcal{S}$. This proves that $\left(\mathfrak{X}_{\boldsymbol{\omega}}\right)_{Q} \subset \mathfrak{S}$, and hence $\left(\mathfrak{X}_{\boldsymbol{\omega}}\right)_{Q} \subset \mathfrak{S}_{1}$ and $\left(\mathfrak{X}_{\boldsymbol{\omega}}\right)_{Q} \subset \mathfrak{S}_{2}$. 
$\mathfrak{S}_{2} \subset\left(\mathfrak{X}_{\boldsymbol{\omega}}\right)_{Q}$ : Conversely, given $\rho \in \mathfrak{S}_{2}$, we can prove that:

(BQ1) For all $\gamma \in \mathcal{S}_{2}$, we have $\operatorname{tr}(\rho(\gamma)) \notin[-2,2]$.

(BQ2) For all $K>0$, the set $\left\{\gamma \in \mathcal{S}_{2}|| \operatorname{tr}(\rho(\gamma)) \mid<K\right\}$ is finite.

We check condition (BQ1) contrapositively: suppose $\gamma \in \mathcal{S}_{2}$ satisfies $\operatorname{tr}(\rho(\gamma)) \in$ $[-2,2]$; then $\rho \notin \mathfrak{S}_{2}$, because we can find a sequence of elements in $\mathcal{S}_{2}$, such that the word length increases exponentially, but the length increases at most quadratically. To do that, we can consider faces that share an edge with the face corresponding to $\gamma$, and use parts (1) and (2) of Lemma 3.9. On the other hand, if there exist $K>0$ such that $\left\{\gamma \in \mathcal{S}_{2}|| \operatorname{tr}(\rho(\gamma)) \mid<K\right\}$ is infinite, then, again $\rho \notin \mathfrak{S}_{2}$, because for any $K>0$ there is only a finite number of elements with word length less than or equal to $K$. This proves $\mathfrak{S}_{2} \subset\left(\mathfrak{X}_{\boldsymbol{\omega}}\right)_{Q}$, so $\left(\mathfrak{X}_{\boldsymbol{\omega}}\right)_{Q}=\mathfrak{S}=\mathfrak{S}_{2}$. Now using Proposition 4.5, we can also conclude that $\mathfrak{S}=\mathfrak{S}_{1}=\mathfrak{S}_{2}=\left(\mathfrak{X}_{\boldsymbol{\omega}}\right)_{Q}$.

An easy corollary of this result is the inclusion $\mathfrak{X}_{\mathrm{ps}} \subset \mathfrak{X}_{Q}$. Indeed, the set $\mathfrak{X}_{\mathrm{ps}}$ corresponds to representations such that the axes corresponding to primitive elements are uniform quasi-geodesics, that is,

$$
\begin{aligned}
& \mathfrak{X}_{\mathrm{ps}}=\{\rho \in \mathfrak{X} \mid \exists k=k(\rho), \\
&\left.\forall \gamma \text { primitive } \forall \gamma_{1}, \gamma_{2} \in \operatorname{Ax}(\gamma),\left|\mathrm{L}\left(\rho\left(\gamma_{1}^{-1} \gamma_{2}\right)\right)\right| \geq k \mathrm{~W}\left(\gamma_{1}^{-1} \gamma_{2}\right)\right\},
\end{aligned}
$$

where $\operatorname{Ax}(\gamma)$ is the axis passing through the identity $e$. Now, taking $\gamma_{1}=e, \gamma_{2}=\gamma$ and noticing that all elements in $\mathcal{S}$ are primitive, we can prove the above inclusion. To see that this inclusion is proper, we can note that the holonomy of a hyperbolic structure with punctures at the three boundary components gives a representation in $\left(\mathfrak{X}_{\boldsymbol{\omega}}\right)_{Q}$, but not in $\mathfrak{X}_{\mathrm{ps}}$. So we have the following.

Proposition 4.13. $\mathfrak{X}_{\mathrm{ps}} \subsetneq \mathfrak{X}_{Q}$.

Finally, we prove the following alternative characterization of Bowditch maps in terms of the attracting subtree.

Proposition 4.14. Let $K \geq 2+M$. Then $\psi \in\left(\boldsymbol{\Psi}_{\boldsymbol{\omega}}\right)_{Q}$ if and only if the tree $T_{\psi}(K)$ is finite.

Proof. Let $K \geq 2+M$. Suppose $\psi \in\left(\mathbf{\Psi}_{\boldsymbol{\omega}}\right)_{Q}$; then the set $\Upsilon_{\psi}^{(2)}(K)$ is finite, and for each element $\xi \in \Upsilon_{\psi}^{(2)}(K)$, we have $\psi(\xi) \notin[-2,2]$ and $\sigma(\xi) \neq 0$. Hence, the function $H_{\psi}(\xi)$ is proper, which implies that the subarc $J_{\psi}(K, \xi)$ is finite for all $K \geq 2+M$. The tree $T_{\psi}(K)$ is now a finite union of finite subarcs.

Now suppose $\psi \notin\left(\boldsymbol{\Psi}_{\boldsymbol{\omega}}\right)_{Q}$. For a fixed $K$ we have three cases:

(1) There exists $\xi$ such that $\psi(\xi) \in[-2,2]$. In this case $\xi \in \Upsilon_{\psi}^{(2)}(K)$, and the arc $J_{\psi}(K, \xi)=\xi$ is infinite. So $T_{\psi}(K)$ is infinite.

(2) The set $\Upsilon_{\psi}^{(2)}(K)$ is infinite. As for each $\xi \in \Upsilon_{\psi}^{(2)}(K)$, the arc $J_{\psi}(K, \xi)$ contains at least one edge, then the tree $T_{\psi}(K)$ is infinite.

(3) There exists $\xi$ such that $\sigma(\xi)=0$. In this case, either $e \in \Upsilon_{\psi}^{(2)}(K)$, in which case the arc $J_{\psi}(K, \xi)=\xi$ is contained in $T_{\psi}(K)$, or $\Upsilon_{\psi}^{(2)}(K)$ is infinite.

In either case, the tree $T_{\psi}(K)$ is infinite, which concludes the proof of the proposition. 
4.3. Openness and proper discontinuity. First we prove the following lemma which will imply the openness of $\left(\boldsymbol{\Psi}_{\boldsymbol{\omega}}\right)_{Q}$.

Lemma 4.15. Let $K>2+M$. For each $\psi \in\left(\boldsymbol{\Psi}_{\boldsymbol{\omega}}\right)_{Q}$, if $T_{\psi}(K)$ is nonempty, there exists a neighborhood $U_{\psi}$ of $\psi \in \mathbf{\Psi}_{\boldsymbol{\omega}}$ such that for all $\phi \in U_{\psi}$, the tree $T_{\phi}(K)$ is contained in $T_{\psi}(K)$.

Proof. Let $\psi \in\left(\boldsymbol{\Psi}_{\boldsymbol{\omega}}\right)_{Q}$, and $K>2+M$ such that $T_{\psi}(K)$ is nonempty. First, it is easy to see that for $\phi$ close enough to $\psi$, the trees $T_{\psi}(K)$ and $T_{\phi}(K)$ have nonempty intersection.

Now, consider the set $E$ of edges which meet $T_{\psi}(K)$ in a single point. Let $e \in E$. We can show that, if $\phi \in \boldsymbol{\Psi}_{\boldsymbol{\omega}}$ is close enough to $\psi$, then $e \notin T_{\phi}(K)$. Indeed, from Lemma 3.14, we have that:

$$
e=\{\alpha, \beta, \gamma\} \notin T_{\phi}(K) \Leftrightarrow
$$

$$
\forall \text { pairwise distinct } x, y, z \in\{\alpha, \beta, \gamma\}, \begin{cases} & |\phi(x)|>K \text { and }|\phi(y)|>K \\ \text { or } & \left|\phi\left(\xi_{x, y}\right)\right|>K^{2}+M \\ \text { or } & |\phi(z)|>H_{\psi}^{*}\left(\xi_{x, y}, K\right) .\end{cases}
$$

On the other hand, since the edge $e$ is not in $T_{\psi}(K)$, we know that one of those strict inequalities holds for $\psi$. So if we choose $\phi$ close enough, the corresponding inequality in $\phi$ will also hold and hence $e \notin T_{\phi}(K)$.

Since the tree $T_{\psi}(K)$ is finite, there is only a finite number of edges in $E$. So again, we can choose $\phi$ close enough so that any edge $e \in E$ is not in $T_{\phi}(K)$.

Now the tree $T_{\phi}(K)$ is connected and $T_{\psi}(K) \cap T_{\phi}(K)$ is nonempty, hence $T_{\phi}(K)$ is entirely contained in $T_{\psi}(K)$.

Theorem 4.16. The set $\left(\boldsymbol{\Psi}_{\boldsymbol{\omega}}\right)_{Q}$ is open in $\boldsymbol{\Psi}_{\boldsymbol{\omega}}$ and the action of $\Gamma$ is properly discontinuous.

Proof. The previous lemma directly implies openness. Indeed, let $\psi \in\left(\boldsymbol{\Psi}_{\boldsymbol{\omega}}\right)_{Q}$ and $K>2+M$ such that $T_{\psi}(K)$ is nonempty. Then for each $\phi$ in the open neighborhood $U_{\psi}$ constructed in the previous lemma, the tree $T_{\phi}(K)$ is contained in $T_{\psi}(K)$ and hence is finite, which proves that $U_{\psi} \subset\left(\mathbf{\Psi}_{\boldsymbol{\omega}}\right)_{Q}$.

To prove that the action is properly discontinuous we take $C$ to be a compact subset in $\left(\boldsymbol{\Psi}_{\boldsymbol{\omega}}\right)_{Q}$. We want to prove that the set

$$
\Gamma_{0}=\{g \in \Gamma \mid g C \cap C \neq \emptyset\}
$$

is finite.

Let $K>2+M$ be such that for any $\psi \in C$, the tree $T_{\psi}(K)$ is nonempty. Now around each element of $C$, there exists a neighborhood $U_{\psi}$ given by Lemma 4.15 . So the set $\left(U_{\psi}\right)_{\psi \in C}$ is an open cover of $C$. We take a finite subcover $\left(U_{\psi_{i}}\right)_{i \in I}$ where $I$ is a finite set.

Now for each element $\psi_{i}$ we take the tree $T_{\psi_{i}}(K)$, and consider the union

$$
\mathcal{T}=\bigcup_{i \in I} T_{\psi_{i}}(K)
$$

By construction, for each element $\phi \in C$, the tree $T_{\phi}(K)$ is contained in $\mathcal{T}$. The forest $\mathcal{T}$ is a finite union of finite trees and hence is itself finite. It follows that the set

$$
\Gamma_{1}=\{g \in \Gamma \mid g \mathcal{T} \cap \mathcal{T} \neq \emptyset\}
$$


is finite. As $T_{g \phi}(K)=g T_{\phi}(K)$, then $\Gamma_{0} \subset \Gamma_{1}$, and hence $\Gamma_{0}$ is finite. This proves that the action is properly discontinuous.

\section{Concluding Remarks}

5.1. Generalization to other surfaces. In this paper we are discussing the existence of a domain of discontinuity for the $\mathrm{n}$ of the mapping class group $\mathcal{M C G}\left(N_{1,3}\right)$ of the three-holed projective plane on $\mathfrak{X}$. A natural idea is to generalize this theory to the case of a general orientable surface $\Sigma_{g, b}$ or nonorientable ones $N_{g, b}$. It turns out that the different characterizations of the Bowditch set $\mathfrak{X}_{Q}$ given in Section 4.2 give us a way to define the Bowditch set $\mathfrak{X}_{Q}\left(\Sigma_{g, b}\right)$ as follows:

$$
\mathfrak{X}_{Q}\left(\Sigma_{g, b}\right)=\left\{\rho \in \mathfrak{X}\left(F_{2 g+b-1}\right)|\exists k=k(\rho),| \mathrm{L}(\rho(\gamma)) \mid \geq k \mathrm{~W}(\gamma) \forall \gamma \in \mathcal{S}\left(\Sigma_{g, b}\right)\right\},
$$

where $\mathcal{S}\left(\Sigma_{g, b}\right)$ is the set of (free homotopy classes of) nontrivial, nonperipheral simple closed curves in $\Sigma_{g, b}$. This definition could also help in understanding the relationship with the set of primitive-stable elements, as we discussed in Proposition 4.13. and likewise, this set can be defined in different ways, see Proposition 4.12.

The problem that arises for this generalization is that the proof of the proper discontinuous action of the mapping class group in the cases until now comes from the simple combinatorial description of the complex of curves in the cases that are considered. Hence it will be interesting to understand the right combinatorial object to use (replacing the complex $\Upsilon$, dual to the complex of curves of $N$ ), because that will allow a generalization of most of the results included here. Note that in the cases analyzed, the surfaces have "low" complexity (in the sense that the number of curves in a pants decomposition is small), the (2-sided) simple closed curves are in correspondence with pants decompositions, and a vertex of $\Upsilon$ (or $\Sigma$ in [2], 24] and [19]) corresponds to a triangulation of the surface. Since simple closed curves are useful in trace relations, while triangulations are convenient to define "flips" or involutions, important for the edge relations, one needs to find a combinatorial object incorporating both these two aspects.

5.2. Real characters. It is interesting to focus on real characters, that is, representations of $\pi_{1}(S)$ into one of the two real forms of $\mathrm{SL}(2, \mathbb{C})$, namely $\mathrm{SL}(2, \mathbb{R})$ and $\mathrm{SU}(2)$. Previous work of Goldman [8] and the second author [22] proves that the mapping class group action is ergodic on the relative $\mathrm{SU}(2)$ character varieties for all compact hyperbolic surfaces, orientable or not, with the exception of $N_{3,0}, N_{1,2}$, and $N_{2,1}$. The SL $(2, \mathbb{R})$ case is much more complex, because one can expect to have domains of discontinuity as well as domains where the action is ergodic. In fact, a complete description of the dynamical decomposition of the action is still unknown in general.

The case of the free group $F_{2}$ of rank two has been studied by Goldman [9] and Goldman, McShane, Tan, and Stantchev [11, who gave a complete description of the dynamics of the action of $\operatorname{Out}\left(F_{2}\right)$ on the real characters. In [19], we proved few results about the real case $\mathfrak{X}^{\mathbb{R}}\left(\Sigma_{0,4}\right)$, but it would be interesting to give a complete dynamical decomposition for the mapping class group action on $\mathfrak{X}^{\mathbb{R}}\left(F_{3}\right)$, like in $[9]$. In addition, it would be also interesting to generalize the work of [1] by considering all five surfaces which have $F_{3}$ as their fundamental group: $\Sigma_{0,4}, \Sigma_{1,2}, N_{1,3}, N_{2,2}$, $N_{3,1}$.

In the case of the free group of rank two, the results of [11 prove that the real representations in the domain of discontinuity for the action of $\operatorname{Out}\left(F_{2}\right)$ all come 
from geometric structures on one of the four surfaces with fundamental group $F_{2}$ $\left(\Sigma_{0,3}, \Sigma_{1,1}, N_{1,2}, N_{2,1}\right)$, including hyperbolic structures with conical points. So a very interesting question would be to understand what happens for $F_{3}$.

5.3. Torelli group action. Another follow-up project that we are planning to study is the action of the Torelli group $\mathcal{T}_{n}$ on $\mathfrak{X}$. As explained in the introduction, and in Remark 2.4, the Torelli group $\mathcal{T}_{3}$ is generated by seven involutions whose action on the character variety is easily described. Hence, the two actions studied here and in [19] can be combined to study the action of $\mathcal{T}_{n}$ on $\mathfrak{X}$.

5.4. McShane identities. Finally, as pointed out in [19, it should be possible to describe some new McShane's identities for the four-holed sphere and for the threeholed projective plane. In [14, Huang and Norbury described an interesting identity for the case of the three punctured projective plane. However, the punctured case is easier since the associated equation is very symmetric. So it would be interesting to start looking at surfaces where the boundary components all have the same trace. Note that in the special case $x=y=z=0$, this corresponds to the work of Hu-Tan-Zhang [13.

\section{ApPendix A. Explicit EXPRESSION OF the $H$ FUnCtion}

In this section we give an explicit expression for the function $H$ defined in Section 3.4. We state it in a more general context, where it could be applied to the functions $H$ defined in Tan-Wong-Zhang [24, Hu-Tan-Zhang [13, and Maloni-Palesi-Tan 19]. Note that all functions are defined on the set of faces and take values in $\mathbb{R}$.

Recall that the setting is the one that is found in Section 3.3.1 where we consider the two bi-infinite sequences of neighboring regions around a given face $\xi$. The edge relations on consecutive edges give a recurrence relation for these bi-infinite sequences. As this setting is similar to the situation for the one-holed torus and the four-holed sphere, we state the expression of the $H$ function for sequences satisfying a particular recurrence relation.

Let $(Q, R, S, X) \in \mathbb{C}^{4}$ be parameters, and consider the sequences $\left(y_{n}\right)$ and $\left(z_{n}\right)$ defined by the recurrence relation:

$$
\left(\begin{array}{l}
y_{n+1} \\
z_{n+1}
\end{array}\right)=\left(\begin{array}{cc}
-1 & -X \\
X & X^{2}-1
\end{array}\right)\left(\begin{array}{l}
y_{n} \\
z_{n}
\end{array}\right)+\left(\begin{array}{c}
Q \\
R-X Q
\end{array}\right),
$$

with $y_{0}$ and $z_{0}$ satisfying the equation:

$$
y_{0}^{2}+z_{0}^{2}+X y_{0} z_{0}-Q y_{0}-R z_{0}=S .
$$

Lemma 3.9 implies that there exists a constant $H=H(Q, R, S, X) \in \mathbb{R}_{>0} \cup\{\infty\}$, depending only on the values $(Q, R, S, X)$, such that there are integers $n_{1} \leq n_{2}$ satisfying: $\left|y_{n}\right| \leq H$ if and only if $n_{1} \leq n \leq n_{2}$, and $\left|y_{n}\right|$ is monotonically increasing for $n \geq n_{2}$ and monotonically decreasing for $n \leq n_{1}$.

This constant is finite for $X \notin[-2,2]$, and $Q^{2}+R^{2}-X R Q-S\left(X^{2}-4\right) \neq 0$.

Lemma A.1. $H$ can be chosen as:

$$
H(Q, R, S, X)=\sqrt{|T|}|\lambda|(W+1)+|\eta|,
$$


where $\lambda$ satisfies $\lambda+\lambda^{-1}=X^{2}-2$, and the other terms are defined by:

$$
\begin{aligned}
T & =\frac{Q^{2}+R^{2}-X R Q-S\left(X^{2}-4\right)}{\left(X^{2}-4\right)^{2}}, \\
\eta & =\frac{X R-2 Q}{X^{2}-4}, \\
W & =\frac{|\lambda|}{\sqrt{|T|}(|\lambda|-1)}\left(|\eta|+\sqrt{|\eta|^{2}+|T|\left(|\lambda|-|\lambda|^{-1}\right)}\right) .
\end{aligned}
$$

Proof. From the recurrence relation satisfied by $y_{n}$ and $z_{n}$ we can deduce that:

$$
y_{n}=A \lambda^{n}+B \lambda^{-n}+\eta,
$$

with $A B=T$.

We can assume, without loss of generality, that

$$
\sqrt{|T|}|\lambda|^{-1} \leq|A|,|B| \leq \sqrt{|T|}|\lambda|
$$

(up to reparametrization in $n$ ).

Suppose that $|\lambda|^{n}>W$. Then we have that

$$
|\lambda|^{2 n}-\frac{2|\eta||\lambda|}{\sqrt{T}(|\lambda|-1)}|\lambda|^{n}-|\lambda| \frac{|\lambda|+1}{|\lambda|-1} \geq 0 .
$$

We deduce that

$$
|\lambda|^{2 n+1} \geq \frac{|B|(|\lambda|+1)}{|A|(|\lambda|-1)}+\frac{2|\eta|}{|A|(|\lambda|-1)}|\lambda|^{n+1} .
$$

This can be interpreted as

$$
\left|y_{n+1}\right| \geq|A||\lambda|^{n+1}-|B||\lambda|^{-n-1}-|\eta| \geq|A||\lambda|^{n}+|B||\lambda|^{-n}+|\eta| \geq\left|y_{n}\right| .
$$

On the other hand we have:

$$
\begin{aligned}
\left|y_{n}\right| & \leq\left|A \lambda^{n}+B \lambda^{-n}\right|+|\eta| \\
& \leq \sqrt{|A B|}|\lambda|\left(|\lambda|^{n}+|\lambda|^{-n}\right)+|\eta| \\
& \leq \sqrt{|A B|}|\lambda|\left(|\lambda|^{n}+1\right)+|\eta| .
\end{aligned}
$$

In conclusion, if $\left|y_{n}\right| \geq \sqrt{|A B|}|\lambda|(W+1)+|\eta|=H$, then we have $|\lambda|^{n} \geq W$, and we can apply the previous computations to show that $\left|y_{n+1}\right| \geq\left|y_{n}\right|$.

In a similar way, we can prove that, if $\left|y_{-n}\right| \geq H$, then $\left|y_{-n-1}\right| \geq\left|y_{-n}\right|$.

Remark A.2. We can also define a function $H^{\prime}$ for the sequence $z_{n}$ by permuting the constants $Q$ and $R$. In other words $H^{\prime}(Q, R, S, X)=H(R, Q, S, X)$.

Now we can apply this formula in several cases. We will follow the notation used in the papers where the respective case is discussed.

(1) The one-holed torus $S_{1,1}$ (Bowditch [2], Tan-Wong-Zhang [24]):

We consider a one-holed torus $S_{1,1}$, with boundary trace equal to $\mu$, that is, we consider the relative character variety $\mathfrak{X}_{(a, b, c, d)}\left(S_{0,4}\right)$. Let's fix a face $X$ such that $\phi(X)=x$. We look at the neighbors of $X$. In this case, we can set:

$$
\begin{aligned}
Q & =R=0, \\
X & =x, \\
S & =\mu-x^{2},
\end{aligned}
$$


and the formula becomes

$$
H\left(0,0, \mu-x^{2}, x\right)=\sqrt{\frac{\left|x^{2}-\mu\right|}{\left|x^{2}-4\right|}} \frac{2|\lambda|^{2}}{|\lambda|-1} .
$$

This corresponds exactly to the function used by Tan-Wong-Zhang ([24], Lemma 3.20).

(2) The four-holed sphere $S_{0,4}$ (Maloni-Palesi-Tan [19]):

We consider a four-holed sphere $S_{0,4}$ with boundary traces $(a, b, c, d)$, that is, we consider the relative character variety $\mathfrak{X}_{(a, b, c, d)}\left(S_{0,4}\right)$. Instead of $\Upsilon$, the complex is called $\Sigma$, and is a trivalent simplicial tree in $\mathbb{H}^{2}$, and the set of faces (or of connected components of $\mathbb{H}^{2} \backslash \Sigma$ ) is denoted $\Omega(\Sigma)$. Let's fix a face $X \in \Omega_{1}(\Sigma)$ (of color 1 ) such that $\phi(X)=x$. We look at the neighbors of $X$. The expression of $(Q, R, S, X)$ for regions in $\Omega_{2}(\Sigma)$ or $\Omega_{3}(\Sigma)$ can be found with a similar method. In this case, we can use:

$$
\begin{aligned}
Q & =b c+a d, \\
R & =a c+b d, \\
X & =x, \\
S & =4-a^{2}-b^{2}-c^{2}-d^{2}-a b c d-(a b+c d) x-x^{2} .
\end{aligned}
$$

(3) The three-holed projective plane $N_{1,3}$ (present paper):

We consider a three-holed projective plane $N_{1,3}$ with boundary traces $(x, y, z)$, that is, we consider the relative character variety $\mathfrak{X}_{(x, y, z)}\left(N_{1,3}\right)$. Let's fix a face $\xi=\xi_{(\alpha, \beta)} \in \Upsilon^{(2)}$ such that $\xi=\alpha \cap \beta$ with $\alpha \in \Upsilon_{1}^{(3)}$ and $\beta \in \Upsilon_{2}^{(3)}$ such that $\psi(\alpha)=a$ and $\psi(\beta)=b$. We look at the neighbors of $\xi$. The expression of $(Q, R, S, X)$ for regions in $\Omega_{2}(\Sigma)$ or $\Omega_{3}(\Sigma)$ can be found with a similar method. In this case, we can use:

$$
\begin{aligned}
Q & =y b+a z, \\
R & =y a+z b, \\
X & =a b-x, \\
S & =4-a^{2}-b^{2}-x^{2}-y^{2}-z^{2}-x y z-x a b .
\end{aligned}
$$

The particular case of $x=y=z=0$ can be written:

$$
H(a, b)=\sqrt{\left|\frac{\sigma-\mu}{X^{2}-4}\right| \frac{2|\Lambda|^{2}}{|\Lambda|-1},}
$$

where $\sigma=a^{2}+b^{2}, X=a b$, and $\Lambda^{2}=\lambda$. This corresponds to the function considered by Hu-Tan-Zhang [13, Lemma 3.5] in the case $n=4$.

The formula gives an explicit method to determine if an edge of the 1-skeleton is in the attracting subtree $T_{\phi}(2+M)$. As the tree is connected, one can produce a computer program testing if a Markoff map is in the Bowditch set. This is an important tool if one wants to generate computer pictures of the Bowditch set.

\section{Appendix B. Relation with the Torelli subgroup}

B.1. Representatives of the involutions. In the introduction, we defined seven involutions on the character variety of the free group $F_{3}$ of rank three, which are 
given by seven involutions in $\operatorname{Out}\left(F_{3}\right)$. We can give explicit representatives of the involutions in $\operatorname{Aut}\left(F_{3}\right)$

$$
\begin{array}{ll}
\widetilde{\tau}_{a}:\left(\begin{array}{l}
A \\
B \\
C
\end{array}\right) \mapsto\left(\begin{array}{c}
C B^{-1} A^{-1} C^{-1} B \\
B^{-1} \\
C^{-1}
\end{array}\right), & \widetilde{\tau}_{x}:\left(\begin{array}{l}
A \\
B \\
C
\end{array}\right) \mapsto\left(\begin{array}{c}
A^{-1} \\
C^{-1} B^{-1} C \\
C^{-1}
\end{array}\right), \\
\widetilde{\tau}_{b}:\left(\begin{array}{l}
A \\
B \\
C
\end{array}\right) \mapsto\left(\begin{array}{c}
A C^{-1} B^{-1} A^{-1} C \\
C^{-1}
\end{array}\right), & \widetilde{\tau}_{y}:\left(\begin{array}{l}
A \\
B \\
C
\end{array}\right) \mapsto\left(\begin{array}{c}
A^{-1} \\
B^{-1} \\
A^{-1} C^{-1} A
\end{array}\right), \\
\tilde{\tau}_{c}:\left(\begin{array}{l}
A \\
B \\
C
\end{array}\right) \mapsto\left(\begin{array}{c}
A \\
B^{-1} \\
B A^{-1} C^{-1} B^{-1} A
\end{array}\right), & \widetilde{\tau}_{z}:\left(\begin{array}{l}
B \\
C
\end{array}\right) \mapsto\left(\begin{array}{c}
B^{-1} A^{-1} B \\
B^{-1} \\
C^{-1}
\end{array}\right), \\
\widetilde{\tau}_{d}:\left(\begin{array}{l}
A \\
B \\
C
\end{array}\right) \mapsto\left(\begin{array}{l}
A^{-1} \\
B^{-1} \\
C^{-1}
\end{array}\right) .
\end{array}
$$

These are representatives of maps $\tau_{a}, \ldots, \tau_{z}$ in $\operatorname{Out}\left(F_{3}\right)$. The action of $\tau_{a}, \ldots, \tau_{z}$ on the character variety $X\left(F_{3}\right)$ is exactly given by the seven involutions $\theta_{a}, \ldots, \theta_{z}$ defined in the introduction.

B.2. Torelli subgroup. There is a natural surjective homomorphism from $\operatorname{Out}\left(F_{n}\right)$ to $\operatorname{GL}(n, \mathbb{Z})$, given by abelianizing the free group $F_{n}$. Its kernel is known as the Torelli subgroup of $\operatorname{Out}\left(F_{n}\right)$, and we denote it by $\mathcal{T}_{n}$ :

$$
1 \rightarrow \mathcal{T}_{n} \rightarrow \operatorname{Out}\left(F_{n}\right) \rightarrow \operatorname{GL}(n, \mathbb{Z}) \rightarrow 1 .
$$

We can also define the subgroup $\mathcal{T}_{n}^{\prime}$ as the inverse image of the center $\left\{ \pm I_{n}\right\}$ by the abelianization map:

$$
1 \rightarrow \mathcal{T}_{n}^{\prime} \rightarrow \operatorname{Out}\left(F_{n}\right) \rightarrow \operatorname{PGL}(n, \mathbb{Z}) \rightarrow 1
$$

It is clear that $\mathcal{T}_{n}$ is an index 2 subgroup of $\mathcal{T}_{n}^{\prime}$. This group is generated by $\mathcal{T}_{n}$ together with the involution $\left(A_{1}, \ldots, A_{n}\right) \mapsto\left(A_{1}^{-1}, \ldots, A_{n}^{-1}\right)$.

Proposition B.1. The group $\Gamma$ generated by the seven involutions $\tau_{a}, \ldots, \tau_{z}$ is equal to $\mathcal{T}_{3}^{\prime}$

Proof. First, it is clear that each involution $\tau_{a}, \ldots, \tau_{z}$ is an element of $\mathcal{T}_{n}^{\prime}$. It remains to show that the generators of $\mathcal{T}_{n}^{\prime}$ can be written as a product of the involutions. We use the Magnus generating set of $\mathcal{T}_{n}$ (see Bestvina-Bux-Margalit [1]) given by their lift in $\operatorname{Aut}\left(F_{n}\right)$ : for distinct $i, j$, and $k$

$$
K_{i j}:=\left(\begin{array}{c}
A_{1} \\
\vdots \\
A_{i} \\
\vdots \\
A_{n}
\end{array}\right) \mapsto\left(\begin{array}{c}
A_{1} \\
\vdots \\
A_{j} A_{i} A_{j}^{-1} \\
\vdots \\
A_{n}
\end{array}\right) K_{i j k}:=\left(\begin{array}{c}
A_{1} \\
\vdots \\
A_{i} \\
\vdots \\
A_{n}
\end{array}\right) \mapsto\left(\begin{array}{c}
A_{1} \\
\vdots \\
A_{i}\left[A_{j}, A_{k}\right] \\
\vdots \\
A_{n}
\end{array}\right) .
$$

We have $K_{i j k}=K_{i k j}^{-1}$ in $\operatorname{Out}\left(F_{n}\right)$, and, when $n=3$, we have $K_{i j}=\left(K_{k j}\right)^{-1}$ in $\operatorname{Out}\left(F_{3}\right)$. So we have the six generators for $\mathcal{T}_{3}$ given by:

$$
K_{12}, K_{23}, K_{31}, K_{123}, K_{231}, K_{312} \text {. }
$$


These six generators together with $\tau_{d}$ generate $\mathcal{T}_{3}^{\prime}$. These generators can now be obtained as products of the involutions as follows:

$$
\begin{aligned}
K_{12} & =\tau_{z} \circ \tau_{d}, \\
K_{23} & =\tau_{x} \circ \tau_{d}, \\
K_{31} & =\tau_{y} \circ \tau_{d}, \\
K_{123} & =\tau_{d} \circ \tau_{x} \circ \tau_{a} \circ \tau_{z}, \\
K_{231} & =\tau_{d} \circ \tau_{y} \circ \tau_{b} \circ \tau_{x}, \\
K_{312} & =\tau_{d} \circ \tau_{z} \circ \tau_{c} \circ \tau_{y} .
\end{aligned}
$$

This proves that the two groups $\Gamma$ and $\mathcal{T}_{3}^{\prime}$ are equal.

\section{ACKNOWLEDGMENT}

The authors would like to thank the anonymous referee for the very careful reading of the manuscript and the many helpful comments and suggestions which greatly improved the presentation.

\section{REFERENCES}

[1] Mladen Bestvina, Kai-Uwe Bux, and Dan Margalit, The dimension of the Torelli group, J. Amer. Math. Soc. 23 (2010), no. 1, 61-105, DOI 10.1090/S0894-0347-09-00643-2. MR2552249

[2] B. H. Bowditch, Markoff triples and quasi-Fuchsian groups, Proc. London Math. Soc. (3) 77 (1998), no. 3, 697-736, DOI 10.1112/S0024611598000604. MR1643429

[3] Richard Canary, Dynamics on character varieties: a survey, Handbook of group actions. Vol. II, 2015, pp. 175-200.

[4] Richard D. Canary and Aaron D. Magid, Dynamics on PSL $(2, \mathbb{C})$-character varieties: 3manifolds with toroidal boundary components, Groups Geom. Dyn. 9 (2015), no. 1, 149-185, DOI 10.4171/GGD/309. MR3343350

[5] Richard D. Canary and Peter A. Storm, Moduli spaces of hyperbolic 3-manifolds and dynamics on character varieties, Comment. Math. Helv. 88 (2013), no. 1, 221-251, DOI 10.4171/CMH/284. MR3008919

[6] Marc Culler and Peter B. Shalen, Varieties of group representations and splittings of 3manifolds, Ann. of Math. (2) 117 (1983), no. 1, 109-146. MR683804

[7] Robert Fricke and Felix Klein, Vorlesungen über die Theorie der automorphen Funktionen. Band 1: Die gruppentheoretischen Grundlagen. Band II: Die funktionentheoretischen Ausführungen und die Andwendungen (German), Bibliotheca Mathematica Teubneriana, Bände 3, vol. 4, Johnson Reprint Corp., New York; B. G. Teubner Verlagsgesellschaft, Stuttg art, 1965. MR0183872

[8] William M. Goldman, Ergodic theory on moduli spaces, Ann. of Math. (2) 146 (1997), no. 3, 475-507, DOI 10.2307/2952454. MR1491446

[9] William M. Goldman, The modular group action on real SL(2)-characters of a one-holed torus, Geom. Topol. 7 (2003), 443-486, DOI 10.2140/gt.2003.7.443. MR2026539

[10] William M. Goldman, Mapping class group dynamics on surface group representations, Problems on mapping class groups and related topics, Proc. Sympos. Pure Math., vol. 74, Amer. Math. Soc., Providence, RI, 2006, pp. 189-214, DOI 10.1090/pspum/074/2264541. MR.2264541

[11] William Goldman, Greg McShane, George Stantchev, and Ser Peow Tan, Automorphisms of two-generator free groups and spaces of isometric actions on the hyperbolic plane, Mem. Amer. Math. Soc. 259 (2019), no. 1249, vii+78, DOI 10.1090/memo/1249. MR3941854

[12] Olivier Guichard and Anna Wienhard, Anosov representations: domains of discontinuity and applications, Invent. Math. 190 (2012), no. 2, 357-438, DOI 10.1007/s00222-012-0382-7. MR.2981818

[13] Hengnan Hu, Ser Peow Tan, and Ying Zhang, Polynomial automorphisms of $\mathbb{C}^{n}$ preserving the Markoff-Hurwitz polynomial, Geom. Dedicata 192 (2018), 207-243, DOI 10.1007/s10711017-0235-z. MR 3749429 
[14] Yi Huang and Paul Norbury, Simple geodesics and Markoff quads, Geom. Dedicata 186 (2017), 113-148, DOI 10.1007/s10711-016-0182-0. MR3602888

[15] François Labourie, Anosov flows, surface groups and curves in projective space, Invent. Math. 165 (2006), no. 1, 51-114, DOI 10.1007/s00222-005-0487-3. MR2221137

[16] Michelle Lee, Dynamics on the $\operatorname{PSL}(2, \mathbb{C})$-character variety of a compression body, Algebr. Geom. Topol. 14 (2014), no. 4, 2149-2179, DOI 10.2140/agt.2014.14.2149. MR3331612

[17] Michelle Lee, Dynamics on the $\operatorname{PSL}(2, \mathbb{C})$-character variety of a twisted I-bundle, Groups Geom. Dyn. 9 (2015), no. 1, 187-201, DOI 10.4171/GGD/310. MR3343351

[18] Alexander Lubotzky and Andy R. Magid, Varieties of representations of finitely generated groups, Mem. Amer. Math. Soc. 58 (1985), no. 336, xi+117, DOI 10.1090/memo/0336. MR818915

[19] Sara Maloni, Frédéric Palesi, and Ser Peow Tan, On the character variety of the four-holed sphere, Groups Geom. Dyn. 9 (2015), no. 3, 737-782, DOI 10.4171/GGD/326. MR3420542

[20] Sara Maloni, Frédéric Palesi, and Tian Yang, On type-preserving representations of thrice punctured projective plane group, 2019. To appear in Journal of Differential Geometry.

[21] Yair N. Minsky, On dynamics of Out $\left(F_{n}\right)$ on $\mathrm{PSL}_{2}(\mathbb{C})$ characters, Israel J. Math. 193 (2013), no. 1, 47-70, DOI 10.1007/s11856-012-0086-0. MR.3038545

[22] Frederic Palesi, Ergodic actions of mapping class groups on moduli spaces of representations of non-orientable surfaces, Geom. Dedicata 151 (2011), 107-140, DOI 10.1007/s10711-0109522-7. MR 2780741

[23] Martin Scharlemann, The complex of curves on nonorientable surfaces, J. London Math. Soc. (2) 25 (1982), no. 1, 171-184, DOI 10.1112/jlms/s2-25.1.171. MR645874

[24] Ser Peow Tan, Yan Loi Wong, and Ying Zhang, Generalized Markoff maps and McShane's identity, Adv. Math. 217 (2008), no. 2, 761-813. MR2370281 (2008k:57035)

[25] Tian Yang, On type-preserving representations of the four-punctured sphere group, Geom. Topol. 20 (2016), no. 2, 1213-1255, DOI 10.2140/gt.2016.20.1213. MR3493103

Department of Mathematics, University of Virginia, Kerchof Hall, Charlottesville, VIRGINIA 22904-4137

Email address: sm4cw@virginia.edu

Aix Marseille Université, CNRS, Centrale Marseille, I2M, UMR 7373, 13453 MarSEILlE, FRANCE

Email address: frederic.palesi@univ-amu.fr 